Nonlinear Processes in Geophysics (2001) 8: 313-330

Nonlinear Processes

in Geophysics

(C)European Geophysical Society 2001

\title{
A study of magnetic fluctuations and their anomalous scaling in the solar wind: the Ulysses fast-latitude scan
}

\author{
C. Pagel and A. Balogh \\ The Blackett Laboratory, Imperial College, London, UK
}

Received: 14 November 2001 - Revised: 2 April 2001 - Accepted: 14 May 2001

\begin{abstract}
The solar wind is a highly turbulent and intermittent medium at frequencies between $10^{-4}$ and $10^{-1} \mathrm{~Hz}$. Power spectra are used to look at fluctuations in the components of the magnetic field at high frequencies over a wide range of latitudes. Results show steady turbulence in the polar regions of the Sun and a more varied environment in the equatorial region. The magnetic field fluctuations exhibit anomalous scaling at high frequencies. Various models have been proposed in an attempt to better understand the scaling nature of such fluctuations in neutral fluid turbulence. We have used the Ulysses fast latitude scan data to perform a wide ranging comparison of three such models on the solar wind magnetic field data: the well-known $\mathrm{P}$ model, in both its Kolmogorov and Kraichnan forms, the lognormal cascade model and a model adapted from atmospheric physics, the $\mathrm{G}$ infinity model. They were tested by using fits to graphs of the structure function exponents $\mathrm{g}(\mathrm{q})$, by making a comparison with a non-linear measure of the deviation of $\mathrm{g}(\mathrm{q})$ from the non-intermittent straight line, and by using extended self similarity technique, over a large range of heliolatitudes. Tests of all three models indicated a high level of intermittency in the fast solar wind, and showed a varied structure in the slow wind, with regions of apparently little intermittency next to regions of high intermittency, implying that the slow wind has no uniform origin. All but one of the models performed well, with the lognormal and Kolmogorov P model performing the best over all the tests, indicating that inhomogeneous energy transfer in the cascade is a good description. The Kraichnan model performed relatively poorly, and the overall results show that the Kraichnan model of turbulence is not well supported over the frequency and distance ranges of our data set. The $\mathrm{G}$ infinity model fitted the results surprisingly well and showed that there may very well be important universal geometrical aspects of intermittency over many physical systems.
\end{abstract}

Correspondence to: C. Pagel (c.pagel@ic.ac.uk)

\section{Introduction}

Kolmogorov's (1991a) derived hypotheses for a homogeneous, isotropic flow (K41 theory) form the basis of a large body of research into the statistical characteristics of turbulent systems. More recently, it has become clear that the velocity fluctuations in many turbulent flows do not scale as predicted by K41 theory, i.e. they show anomalous scaling (see Frisch, 1995, for a comprehensive fluid dynamical review). Such systems are then often described as exhibiting intermittent turbulence. However, the causes or mechanisms for such anomalous scaling are far from clear, and there exist several models or theories attempting to achieve a better understanding of such flows. One popular approach is that of generating intermittency via an inhomogeneous energy transfer cascade, with different models distributing energy differently through the cascade. These models are then tested against available data (for instance, Meneveau and Sreenivasan (1987a,b); Borgas (1992) for neutral fluids and Ruzmaikin et al. (1995); Marsch and Tu (1997); Horbury and Balogh (1998) for the solar wind). We will use two such models in this paper (see Sect. 5.1). Currently, there is no dominant model, so there is no consistent way of quantifying intermittency in a system.

The defining parameter controlling turbulence in a flow is the Reynolds number, defined as the ratio of inertial to viscous forces, $L V / v$, where $L$ and $V$ are the characteristic length scale and velocity of the fluid and $v$ its kinematic viscosity. The solar wind is an environment particularly suited to studying these phenomena since it displays fully developed intermittent turbulence at higher Reynolds number than can be achieved in laboratory flows: the highest recorded laboratory Reynolds numbers so far is 23000 , achieved at the National Aerospace Facility whereas, with a kinematic viscosity approaching zero, the kinematic Reynolds number in the solar wind is approaching infinity. In magnetohydrodynamic (MHD) systems, a magnetic Reynolds number can also be defined, analogous to its counterpart in neutral fluid 


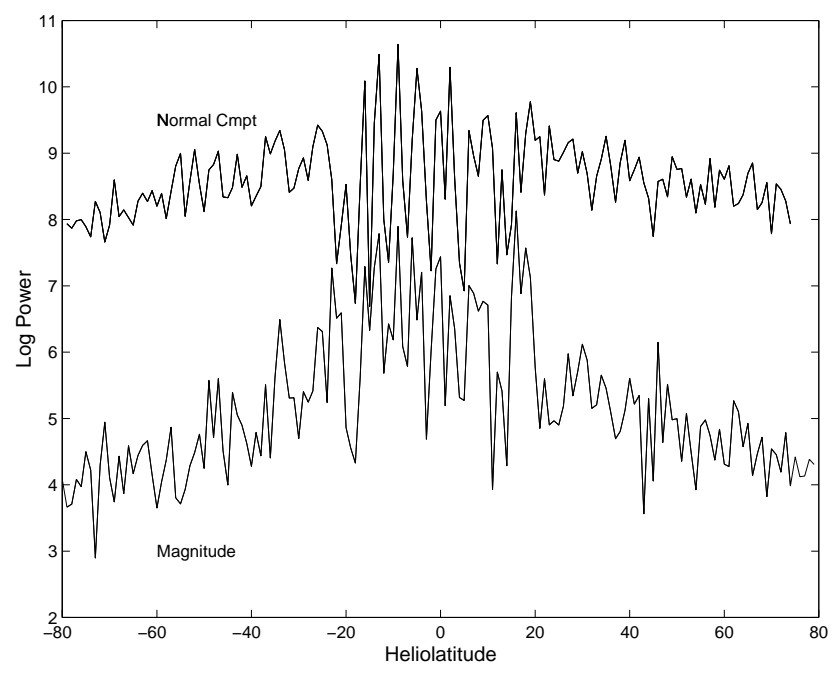

Fig. 1. Comparison of power at $10^{-4} \mathrm{~Hz}$ between the $\mathrm{N}$ component and magnitude of the magnetic field. The power has units $\mathrm{nT}^{2} / \mathrm{Hz}^{2}$. There is a similar difference for the radial and tangential components.

turbulence, as $L V / \eta^{*}$, where $\eta^{*}$ is the magnetic diffusivity. In the solar wind, this parameter is as high as $10^{10}$ (Horbury, 1996). Recent studies into solar wind turbulence have been conducted by Roberts et al. (1987); Kraichnan (1990); Marsch and Liu (1993); Carbone (1993); Feynman and Ruzmaikin (1994); Tu and Marsch (1995); Velli and Prunetti (1997); Horbury et al. (1997b); Horbury and Balogh (1998). With the launch of the Ulysses satellite ten years ago, it has been possible to obtain an extremely accurate picture of the solar wind, in particular, its magnetic field fluctuations over a long period of time and a wide range of heliolatitudes.

Using the statistical techniques associated with turbulence and intermittency on this data set, we hope to further investigate both the nature of these fluctuations in the magnetic field and their scaling behaviour. In Sects. 2 and 3, brief overviews of structure function analysis and the solar wind are given respectively. In Sect. 4, the fast-latitude scan results using power spectral analysis are presented, while Sect. 5 deals with the analysis of intermittency using structure function analysis, and includes a description of the intermittent models used. Sect. 6 analyses the results of Sect. 5 with reference to solar wind speed, and finally, Sect. 7 discusses the conclusions of this study.

\section{Statistical methods for studying fluctuations}

Power spectra are probably the most familiar way of analysing scaling properties in a system, particularly in turbulence. In systems where the fluctuations are Gaussian, they completely characterise their nature, however, in cases where the underlying probability distributions have a different behaviour, higher order moments are needed to characterise the scaling properties of the fluctuating field. In this sense, structure functions provide a better alternative to power spectra, since they pick up on a fuller range of statistics, while still providing the information given by spectra through a formal equivalence of the second order structure function with a power spectrum (see below). A quick overview of structure functions is given. For a more detailed introduction, see Frisch (1995).

A structure function of order $q$, for a time series $x(t)$, is defined as follows:

$S(q, \tau)=\left\langle|x(t+\tau)-x(t)|^{q}\right\rangle$

where $\tau$ represents the time lag between points, and the \langle\rangle represent time averages. Absolute values for the structure functions have been used here since they give better statistical convergence and also ensure that odd and even order functions have the same large-scale saturation behaviour (Vahnstein et al., 1994; Grossmann et al., 1997). Certainly, the use of absolute values is now customary in solar wind intermittent studies (Marsch and Liu, 1993; Ruzmaikin et al., 1995; Tu and Marsch, 1995; Horbury et al., 1997b; Horbury and Balogh, 1998; Veltri, 1999).

In a system exhibiting scaling, structure functions have a power law dependence:

$S(q, \tau) \sim \tau^{g(q)}$.

There is an equivalence between the second order structure function and the power spectrum: if $S(2, \tau)$ has in$\operatorname{dex} g(2)$, then the power spectrum will have index $\alpha$ where $\alpha=-g(2)-1$. If no intermittency is present, then the structure function index is linear with the order, i.e.:

$g(q)=a q$

where $a$ is a constant. In fact, in Kolmogorov turbulence, $a=1 / 3$ (Frisch, 1995). However, in an intermittent system, $g(q)$ flattens out for larger $q$ and linearity is lost.

In classical turbulence, the power spectrum index for the velocity function under isotropic, homogeneous, incompressible fluid conditions is $-5 / 3$, and the corresponding second order structure function index is $2 / 3$ (from Kolmogorov, 1991a,b). This is still largely the case in intermittent systems since the corrections to these values are very small for such low orders of the structure function, but any corrections will increase the absolute value of both the structure function and spectral index.

In both intermittent and non-intermittent turbulence, the third order structure function should theoretically scale with the separation inside the inertial range (the range of frequencies where the dominant process is energy transfer and not energy injection or dissipation):

$S(3, \tau) \sim \tau$.

This relationship has led to the idea of extended selfsimilarity, where instead of plotting the structure functions against $\tau$, they are plotted against the third order structure function instead. This has been hypothesised to give an extended scaling regime for the time series, both further into the dissipation region (Stolovitzky and Sreenivasan, 1993; 
Carbone, 1994; Sain and Bhattacharjee, 1999) and into the large-scale region (Grossmann et al., 1997). This will be considered further in Sect. 5.3.

\section{The solar wind}

Over the last thirty years or so, the solar wind has been extensively studied with the statistical techniques used for neutral fluid turbulence, beginning with Coleman (1968) and Belcher and Davis (1971), whereupon it has been found to be a highly turbulent system. Most of these turbulent fluctuations are thought to be due to Alfvén waves propagating outwards from the sun. For details of the Alfvénic nature of solar wind turbulence, see the reviews by Roberts et al. (1987); Burlaga (1995); Goldstein and Roberts (1995); Tu and Marsch (1995); Bruno (1997), and Goldstein and Roberts (1999). Alfvén waves are the incompressible wave mode of ideal MHD plasmas, and are characterised by:

$\delta \boldsymbol{v} \sim \delta \boldsymbol{B}$

so that the magnetic field fluctuations are in direct correlation with those of the velocity field. This correlation generally holds in the fast solar wind, but breaks down in the slow wind (see, for instance, Roberts et al., 1987). Therefore, the analysis presented here of magnetic fluctuations in the solar wind is not any type of absolute guide to the nature of the velocity fluctuations and the dynamical turbulence. However, the power spectra of the magnetic field at high frequencies indicate a turbulent process and similarly, the structure function analysis highlights the anomalous scaling of these fluctuations (see also Feynman and Ruzmaikin, 1994), so they are worthy of study in their own right.

The Ulysses satellite was launched in 1990 and with a gravity assist from Jupiter, the satellite entered a near polar orbit of the Sun (for more on the Ulysses mission, see Balogh et al., 1992). It is the only satellite to have studied the poles of the Sun and the information it gives, with respect to the range of heliolatitudes it covers, is invaluable. From September 1994 to June 1995, it covered heliolatitudes from $80^{\circ} \mathrm{S}$ to $80^{\circ} \mathrm{N}$, a period known as the fast-latitude scan (Balogh and von Steiger, 2001). The results presented in this paper concentrate on this scan, specifically the vector components of the magnetic field in RTN coordinates, where R is the radial component directed away from the Sun, $\mathrm{T}$ is the tangential component and is the vector product of the Sun's rotation vector with the $\mathrm{R}$ direction, and $\mathrm{N}$ is the normal direction completing the right-hand orthogonal set. It is hoped that an overview of the processes in the solar wind can be gained: most studies so far have concentrated on a few particular sets of data, each lasting from between a few hours to a few days, e.g. Ruzmaikin et al. (1995); Horbury et al. $(1996,1997 b)$. To give a feel for how the solar wind behaviour changes with latitude (at solar minimum), the data was split into segments corresponding to degrees of heliolatitude instead of equal periods of time.
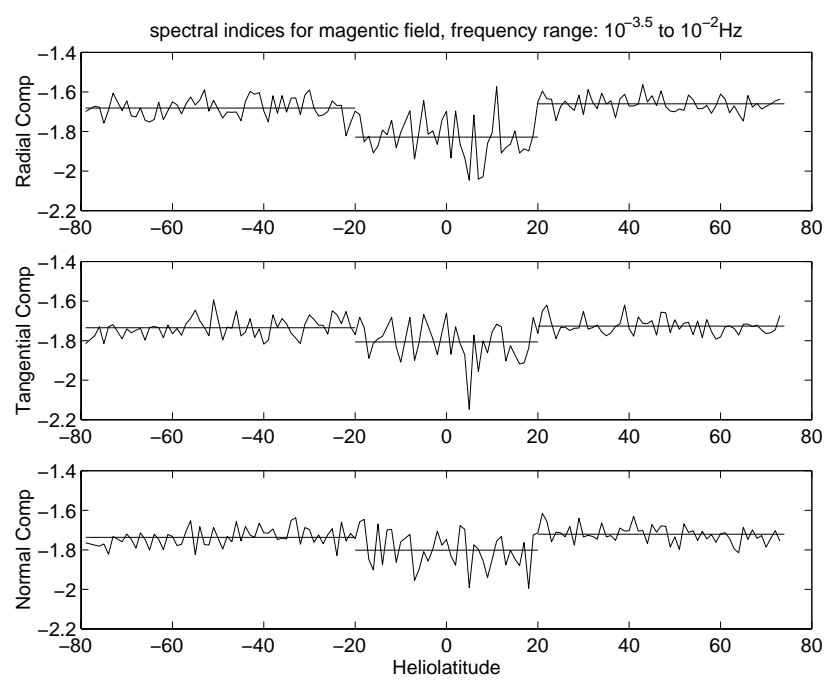

Fig. 2. Spectral indices. The three plots show the power spectral indices for each component of the magnetic field, against heliolatitude. The horizontal lines are drawn at the mean values of the index in the two regions of fast wind, and the slow wind region at the equator.

Note that at these time scales (between 1 and 3 days), solar wind data is normally considered to be weakly stationary (Goldstein and Roberts, 1995; Tu and Marsch, 1995), i.e. the time series increments are dependent only on the time lag between them and not on the time the measurements were taken. However, it should be noted that we did not test each time interval we used for stationarity, since the application of such criteria, in general, is not easy and we are using a wide range of separate time series. Nor did we perform any additional selection on the basis of features in the data, such as shocks. However, there were very few shocks or CMEs recorded over the fast-latitude scan, and more than one shock in an interval would be needed to greatly affect the resulting power spectrum (Matthaeus and Goldstein, 1982). Ulysses data has few data gaps, but those that were there were filled using linear interpolation.

During the fast-latitude scan, Ulysses' distance from the Sun varied between 1.3 and 2.4 AU. At these distances solar wind turbulence is not fully developed, and the inertial range, especially over the polar regions of the Sun, is quite small (Horbury et al., 1996; Horbury and Balogh, 1998). With this in mind, this study is restricted to relatively high frequency observations of the magnetic field, from $10^{-3.5} \mathrm{~Hz}$ to $10^{-2}$ $\mathrm{Hz}$, using power spectra, and a maximum time interval of 300 seconds using structure function analysis. This guarantees that we are firmly inside the inertial range (see also Bavassano et al., 1982; Goldstein and Roberts, 1995, 1999).

\section{Results: power spectra}

The magnetometer data from the fast-latitude scan was analysed per degree of heliolatitude, from $-79^{\circ}$ to $+74^{\circ}$, with a 
Table 1. Spectral index means and their standard errors in the different solar wind regions: southern solar wind from $-79^{\circ}$ to $-19^{\circ}$, equatorial region from $-20^{\circ}$ to $20^{\circ}$ and the northern solar wind from $21^{\circ}$ to $74^{\circ}$

\begin{tabular}{cccc}
\hline Component & S. Wind & Eq. Region & N. Wind \\
\hline $\mathrm{R}$ & -1.68 & -1.83 & -1.66 \\
$\mathrm{~T}$ & -1.74 & -1.81 & -1.73 \\
$\mathrm{~N}$ & -1.74 & -1.80 & -1.72 \\
\hline Component & \multicolumn{3}{c}{ Standard Error in Each Region } \\
\hline $\mathrm{R}$ & 0.004 & 0.008 & 0.003 \\
$\mathrm{~T}$ & 0.004 & 0.008 & 0.003 \\
$\mathrm{~N}$ & 0.004 & 0.008 & 0.004 \\
\hline
\end{tabular}

resolution of 10 seconds. Throughout the scan, the power in the magnitude is much less than in the other components (see Fig. 1), indicating that most of the fluctuations are incompressible and, hence, indeed Alfvénic (Belcher and Davis, 1971; Goldstein and Roberts, 1999). Given the large range of latitudes we are using, this paper will concentrate only on the magnetic field components, and not on the magnetic field magnitude which displays different characteristics. The data from latitudes $-78,-72,-50$ and -9 were discarded since the data over these periods was unreliable. For each degree of heliolatitude, this gives between 8640 and 25920 data points. The power spectra for each of the components of the magnetic field were estimated using a fast-Fourier transform with Thomson multi-tapering (see Percival and Walden (1993) and Denison et al. (1999) for more details on this technqiue). For each degree of heliolatitude, the scaling exponent $\alpha$ was calculated for the inertial range: $E(f) \sim f^{-\alpha}$. The results for the entire fast-latitude scan are shown in Fig. 2.

The fast wind region shows a steady turbulent index, with spectral index slightly higher than the Kolmogorov value, except for the radial component which is very close to $-5 / 3$. The slow wind, however, shows a clear dip in the spectral gradient (see Table 1 for the actual mean values). Bavassano et al. (1982) analysed Helios data in a frequency range of $10^{-4}$ to $10^{-2} \mathrm{~Hz}$ of the magnetic field component, at the edges of high speed streams at low latitudes. They found for data at $0.89 \mathrm{AU}$ an average spectral index of about $-1.65 \pm 0.2$, which, while lower, does not contradict the results presented here.

The central region has a steeper gradient, indicating a somewhat different environment in the equatorial region at these frequencies. Since we are interested in changes in the spectral index, we need some measure of how significant the above dips in the mean index are. To do this, we have taken the average deviation from the mean over the whole range of heliolatitudes, $1 / n \sum_{i=1}^{n}\left|x_{i}-\bar{x}\right|$. We express this as a percentage of the maximum range of the actual index values, $x_{\max }-x_{\min }$, and use it as a base measure of variability. Then, we have averaged the two values of the mean index in the fast wind for each component, and taken the dif-
Table 2. Percentage variation of the spectral index means of the magnetic field components between fast and slow wind compared with the base variation across all heliolatitudes

\begin{tabular}{ccc}
\hline Component & Variability (\%) & Diff. in Means (\%) \\
\hline $\mathrm{R}$ & 15 & 33 \\
$\mathrm{~T}$ & 9 & 14 \\
$\mathrm{~N}$ & 13 & 19 \\
\hline
\end{tabular}

ference between this value and the mean in the slow wind: $\left(m_{f_{1}}+m_{f_{2}}\right) / 2-m_{s}$, where $m_{s}$ is the mean spectral index in the slow wind, and $m_{f_{i}}$ is the mean index in the two regions of fast wind. We calculated this number as a percentage of the maximum range for each component of the magnetic field. The results of this are shown in Table 2, with all percentages rounded to the nearest whole number. The drops seen in the spectral index are significant for all the components of the magnetic field. Tu and Marsch (1995) estimated the spectra of Elsässer variables using Helios data, and in the frequency range $10^{-4}$ to $10^{-3} \mathrm{~Hz}$ found an average index for the radial component of -1.64 in fast wind and of -1.7 in slow wind, for outward propagating Alfvén waves. While this is obviously not the same as looking only at magnetic data as we are doing, it does suggest that there are processes in the equatorial region which act to steepen observed spectral indices.

The transition is particularly evident for the radial component probably because this aspect of the magnetic field is more susceptible to compressive fluctuations in the solar wind which are prevalent in the equatorial region, where most solar activity is taking place. It is also clear that the spectral index of the radial component of the field is significantly different from the perpendicular components of the magnetic field.

The differing behaviour of the high and low frequency contributions to the fluctuations was also analysed (see Fig. 3). This frequency cut-off at $10^{-3.5} \mathrm{~Hz}$ is in agreement with previous results (Bavassano et al., 1982; Smith, 1989; Horbury, 1996; Velli and Prunetti, 1997). In the fast wind, the low frequency spectra have a reasonably stable index of -1 , indicative of the energy-injection scale (Matthaeus and Goldstein, 1986; Smith, 1989; Smith et al., 1995; Horbury et al., 1997a), whereas in the slow wind, the index drops sharply towards -2 . Clearly, the low frequency fluctuations in the solar wind display more variability and also have more power than the high frequency range we have concentrated upon in this study. For more detail on the low frequency region of the fluctuations, see Burlaga's book (1995).

\section{Results: intermittency}

One way of looking for intermittency in the solar wind is to look at the deviation from linear behaviour of a plot of $g(q)$ vs. $q$. An example (see Fig. 4) of one such plot taken in 

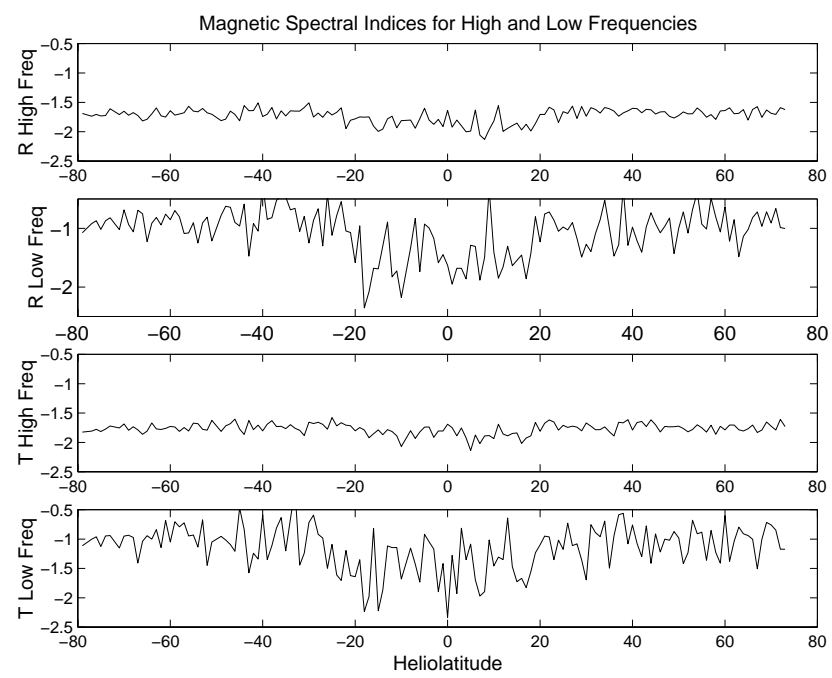

Fig. 3. High and low frequency gradients. Spectral indices for frequencies above $10^{-3.5}$ and below $10^{-3.5} \mathrm{~Hz}$. Only the $\mathrm{R}$ and $\mathrm{T}$ components of the magnetic field are shown here, but the normal component is similar.

the southern polar fast wind shows clear intermittency in the solar wind.

The Ulysses magnetic field data was analysed using structure functions. Time lags of 10 seconds and multiples of 10 seconds were used up to a maximum time lag of $300 \mathrm{sec}-$ onds. Note that using multiples of 10 seconds gives greater detail than the more traditional method of using steps taken in jumps of $2^{n}$ seconds, although admittedly, in our relatively small time range, this is necessary. The range was chosen to keep within the small-scale inertial range identified by Horbury $(1996 ; 1997 b)$. The structure functions up to order five were calculated for each degree of heliolatitude; higher orders would become too unreliable for data sets of the length that we were using (Horbury, 1996; Marsch and Tu, 1997; Horbury and Balogh, 1998). Their scaling index was calculated with respect to the time lag, i.e. $g(q)$ in Equation 2. The data from heliolatitudes $-78,-72,-50$ and -9 has again been discarded.

To try to understand this scaling behaviour, various models have been proposed which attempt to model the fluid processes. These models are based on some hypothesised aspect of the flow, and the predicted structure function index equation for $g(q)$ is calculated and tested against the data. There are two main constraints which need to be built into turbulent intermittency models: firstly, in the limit of zero intermittency, the proposed model for $g(q)$ needs to revert back to the K41 version, namely $g(q)=q / 3$, and secondly, models must fulfill the criteria that $g(3)=1$ (see Eq. 4). The only exception to this rule is if the intermittency model is based on the Kraichnan (1965) modification to K41 theory, namely Kraichnan's attempt to add magnetic field effects to the Kolmogorov fluid theory (KI65 theory). In this case, $g(q)=q / 4$ and $g(4)=1$ are the constraints on the models.

As mentioned above, Horbury and Balogh (1998) high-

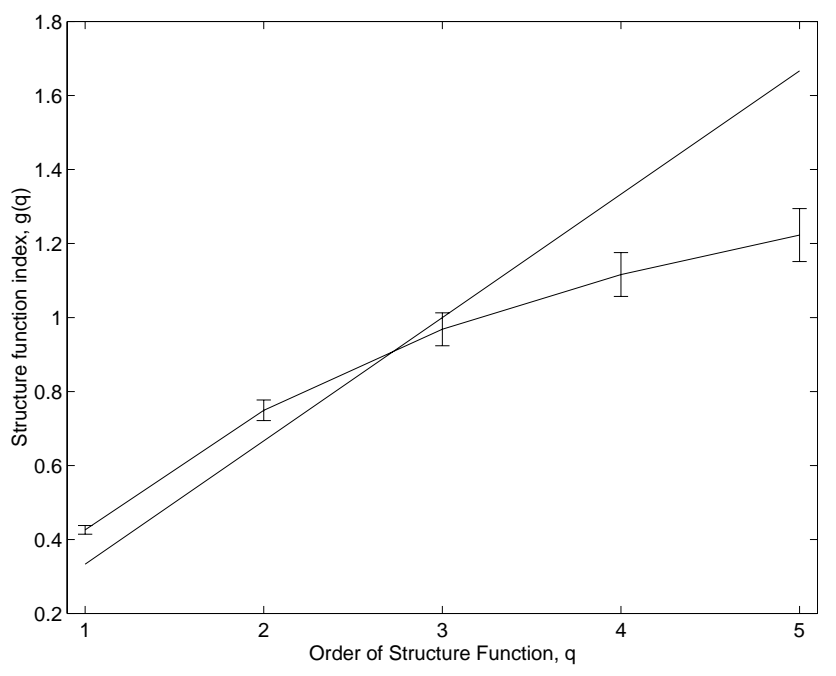

Fig. 4. The straight line in the above graph represents the nonintermittent $g(q)=q / 3$ case, while the curved line shows the $g(q)$ curve for the solar wind, along with associated error bars from fitting a straight line to the individual structure functions of different orders. It is clearly intermittent since it deviates significantly from the non-intermittent linear relationship. Note that the corrections to $g(q)=q / 3$ are positive for $q<3$ and negative for $q \geq 3$, in agreement with Vahnstein et al. (1994).

lighted the problems involved in using structure function analysis with regard to using high order statistics. The data sets used in this study are not long enough to justify using more than five orders of magnitude and so we are attempting to distinguish between different models on the basis of model fits to five data points. It is hoped that studying the fits over the entire range of heliolatitudes will help in this task. For instance a good model should show a relatively stable level of intermittency in the fast wind.

Another measure of the model fits is to look at the intermittency without the use of any model at all. Structure functions and their anomalous scaling have been looked at before, but either models are used to try to quantify the intermittency (see above for examples) or else no quantification is attempted (e.g. Marsch and Liu, 1993). We have tried to introduce such a quantification in order to have something to test the models against on a very basic level. As Marsch and Liu (1993) state, "multifractality is not a directly measurable quantity but merely a mathematical concept". Therefore, an independent measure means a quantification of the non-linearity of the structure function index curve $g(q)$, i.e., its deviation from the non-intermittent $g(q)=q / 3$ case. Since all the models to be studied are tested by their ability to replicate the observed $g(q)$ curves, i.e. the observed deviation from the straight line, using an independent measure of this deviation is a reasonable check to perform on the model fits. To do this, we have used the simplest way of measuring the deviation, where for each degree of heliolatitude, the 'nonlinearity' measure $\sum_{q}(|g(q)-q / 3|)$, the sum of the vertical differences, is used. 


\subsection{Intermittency models}

Three models were studied closely over the whole range of data. It should be noted that these models were not designed to be applied to magnetic field fluctuations, and indeed one model was not meant for any type of fluid data at all. However, given that we have yet to understand the mechanisms behind intermittency in the magnetic field, and the corresponding scarcity of intermittency models formulated for precisely this system, we can make use of intermittency models already available which have proven successful in other systems. As we shall see below, all the models prove relatively successful in modelling the magnetic field fluctuations.

The models were selected on the basis of their goodness of fits over the range of heliolatitudes. On these grounds, the She-Leveque model (SL model) is not included in this paper, as it did not yield good results. It has two forms, a 1D form (6) and a 2D form (7), shown below:

$g(q)=\frac{q}{9}+2\left(1-\left(\frac{2}{3}\right)^{q / 3}\right)$

$g(q)=\frac{q}{9}+1-\left(\frac{1}{3}\right)^{q / 3}$

It also has a Kraichnan form which is not shown here. This model has been used extensively (for example, Politano and Pouquet, 1995; Marsch and Tu, 1997; Horbury and Balogh, 1998; Ching, 2000), and these authors have found the SL model to give good fits, especially the 2D version, which assumes sheet-like structures in the solar wind. The same calculations were made for the SL model as for the other models used in this study. Its fit to the data was consistently worse over the whole range of heliolatitudes than the fits of the other models. A more theoretical consideration is its lack of a free parameter. Given that the model has no such parameter, it essentially assumes uniform intermittency throughout the solar wind. This is akin to saying that all intermittency is the same (once its geometry has been chosen to be 1D or 2D), and that it is an 'on/off' condition, whereby a flow either is intermittent or it is not. However, looking at the data below, this does not seem to be a good description of the solar wind over the whole range of latitudes, where variations in the level of intermittency of the flow are evident. Politano and Pouquet (1995) suggested that the model works well only when far away from the Sun, where the solar wind has become more homogeneous and the turbulence fully developed. It is possible that the model is simply not suitable for such a wide ranging scan relatively close to the Sun. However, despite the above justifications for its exclusion here, the support given to the SL model by others should justify a separate study into the SL model over the fast-latitude scan, but such a study would be too extensive to be included here.

\subsubsection{P models}

We studied two variations of the $\mathrm{P}$ model, one based on K41 theory and the other on the Kraichnan theory (from now on, these two models will be referred to as the PK41 and the PK65 models, respectively). The P model, introduced by Meneveau and Sreenivasan (1987b), uses the framework of the Richardson cascade, where energy is transferred through the scales by the breaking up of larger eddies into smaller eddies and so on. In the K41 framework, the energy given to daughter eddies is equal, making energy distribution throughout the flow homogenous. In the P model, a proportion $p$ of the energy is transferred to one daughter eddy, with $1-p$ going to the other daughter. Clearly, $p=1 / 2$ gives back the $\mathrm{K} 41$ theory, and $p=1$ provides maximum intermittency. So essentially the energy dissipation through the cascade follows a binomial-type distribution with a constant parameter.

The predicted structure function equations for these models are as follows:

K41:

$g(q)=1-\log _{2}\left(p^{q / 3}+(1-p)^{q / 3}\right)$,

KI65:

$g(q)=1-\log _{2}\left(p^{q / 4}+(1-p)^{q / 4}\right)$.

Note that for Eq. 8, $g(3)=1$ while in Eq. 9, $g(4)=1$ and both equations give back the expected non-intermittent linear functions when $p=1 / 2$. This model has proven to be popular and successful over several varied data sets, with many people finding it among the best models available at the moment (Borgas, 1992; Burlaga, 1995; Tu and Marsch, 1995; Horbury, 1996; Marsch and Tu, 1997; Horbury and Balogh, 1998).

\subsubsection{Lognormal model}

This model, introduced by Kolmogorov in 1962 (Frisch, 1995; Burlaga, 1995), also uses the concept of the turbulent cascade, but now the inhomogeneity of energy transfer is not constant throughout the flow but varies with each scale. Here, the energy flux, $\epsilon$, from one scale to the next is multiplied by an independent realisation of a random variable $W$ at each level, where $W$ follows a lognormal distribution, i.e. $W=2^{-m}$ where $m$ is Gaussian, and $\langle W\rangle=1$. Therefore, if at the $1^{\text {st }}$ level $\epsilon=\epsilon_{1}$, then at the $n^{\text {th }}$ level, $\epsilon_{n}=\epsilon_{1} W_{1} \ldots W_{n}$.

The predicted structure function exponents for this model have the following form:

$g(q)=\frac{q}{3}+\frac{\mu}{18}\left(3 q-q^{2}\right)$

where $\mu=0$ gives back the non-intermittent K41 framework. The lognormal model has not proved to be popular, despite giving good fits to data (Burlaga, 1995) since it has two theoretical problems: it violates a theoretical inequality introduced by Novikov (see Frisch (1995) for details) and, often considered more important, it implies supersonic velocities at high Reynolds numbers, not seen in laboratory or atmospheric flows. However, as we are dealing with the solar wind, which is supersonic, the latter is less of a problem 

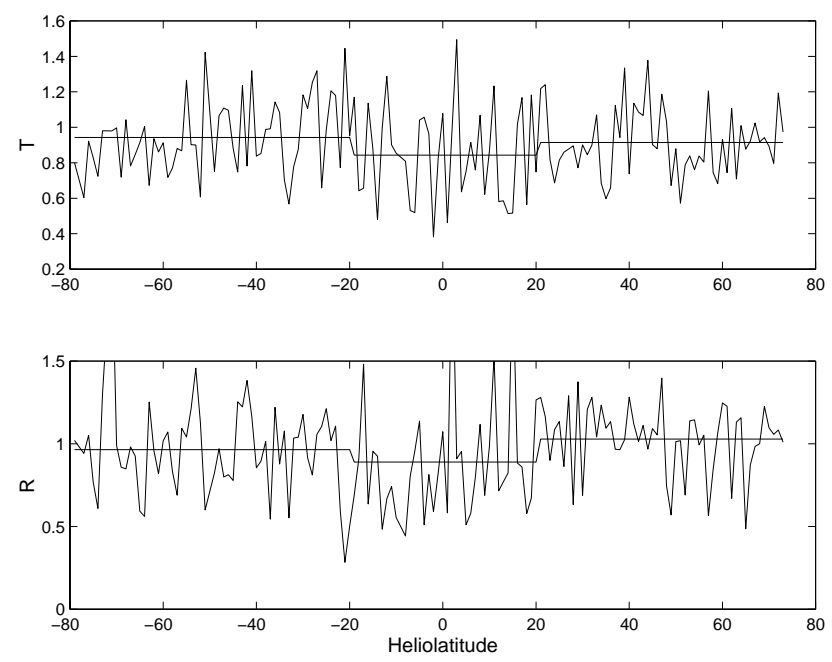

Fig. 5. Nonlinearity, $\sum_{q}(|g(q)-q / 3|)$, of the radial and tangential components of the solar wind magnetic field. Horizontal lines represent means in the two sections of fast wind and in the equatorial region.

in this study, while the precise relevance of the Novikov inequality to the solar wind components of the magnetic field has yet to be considered. Let us also note for the sake of clarity that a spatial lognormal distribution of the magnetic field has been put forward as an explanation for the low-frequency $1 / f$ spectrum (Matthaeus and Goldstein, 1986). However, this is a different issue from the lognormal model proposed here, which deals with the distribution of the energy dissipation through a turbulent cascade.

\subsubsection{G-infinity model}

This model is adapted from one looking at intermittency in atmospheric humidity fluctuations, introduced by Pierrehumbert (1999) and later used by Cho et al. (2000). The original model was not intended for fluid dynamics, and, in particular, not for a turbulent system. Pierrehumbert was looking at self-similarity in cloud variability in the outgoing longwave radiation obtained from satellite measurements. The model he formulated is an empirical model, chosen to best fit the data he was using as opposed to attempting to model the system theoretically. It has the following structure function exponent equation:

$g(q)=\frac{a q}{1+a q / g(\infty)}$

where $a$ is thought to characterise the smoothness of the time series, while $g(\infty)$ characterises the intermittency. Assuming that there are aspects of intermittency which are universal across different systems, we adapted the model above for turbulence by simply adding in the turbulent constraint that $g(3)=1$, reducing to the following one-parameter model:

$g(q)=\frac{g(\infty) q}{3 g(\infty)-3+q}$

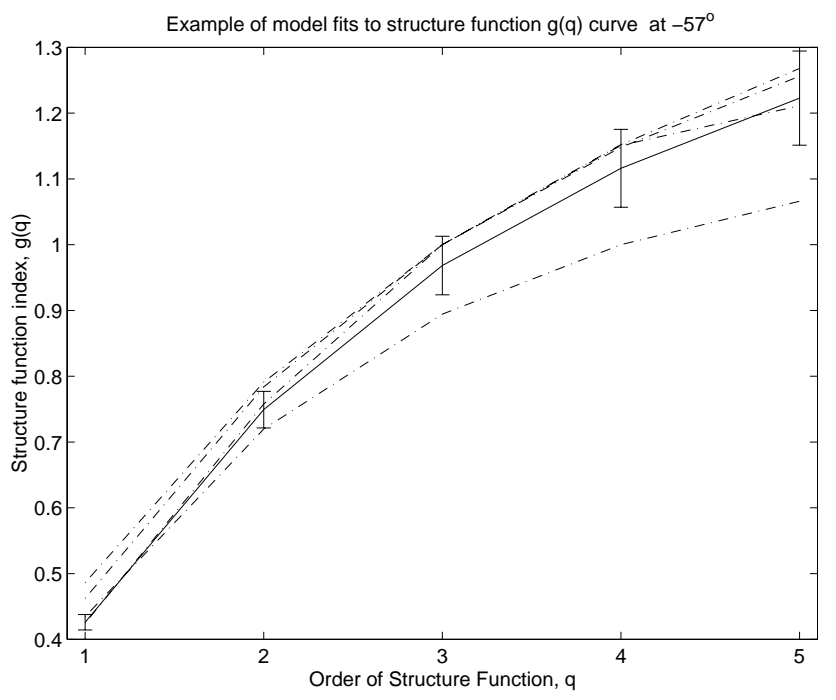

Fig. 6. An example of the 4 model fits to an observed $g(q)$ curve in the fast wind at $-57^{\circ}$. The solid line represents the observed $\mathrm{g}(\mathrm{q})$ curve for structure functions taken with time lags $10 \mathrm{~s}$ to $300 \mathrm{~s}$ with associated error bars. The dashed lines represent the best-fit intermittent model predictions to that curve for the two versions of the P model, lognormal model and G-infinity model.

This conforms to the constraint that $g(q)=q / 3$ when $g(\infty)$ $\rightarrow \infty$ with zero intermittency. We call this model the Ginfinity model for ease of reference, since $g(\infty)$ is the only free parameter. Again, we emphasise that this model is empirical and has no theoretical underpinning, unlike the multiplicative cascade models. Hence, if it were to give a reasonable fit to the data, it could indicate that the dominant process in intermittency is independent of the system it manifests itself in.

\subsection{Model fits}

Before we look at the model fits to the data for each of the models, we first present the results of the non-linearity measure in Fig. 5. All the results in this section are shown for the radial and tangential components of the magnetic field, since the tangential component can be taken as representative of the normal one.

Although the non-linear measure is noisy, there is still a noticeable dip in the mean in the equatorial region, indicating an overall reduction in intermittency in this region. The model fits should mirror this behaviour to some extent, and should also be less noisy.

Each of the models from the previous section was fitted to the structure function index curve for each degree of heliolatitude (Fig. 6). The fits are calculated by varying the free parameter in the model in jumps of 0.01 and then picking the parameter which minimises the least-squares fit value, $\sum_{q}(g(q)-x(q))^{2}$, where $x(q)$ represents the observed value of the structure function indices and $g(q)$ represents the model prediction for those indices. The fits for each degree of heliolatitude are then characterised by the best-fit param- 

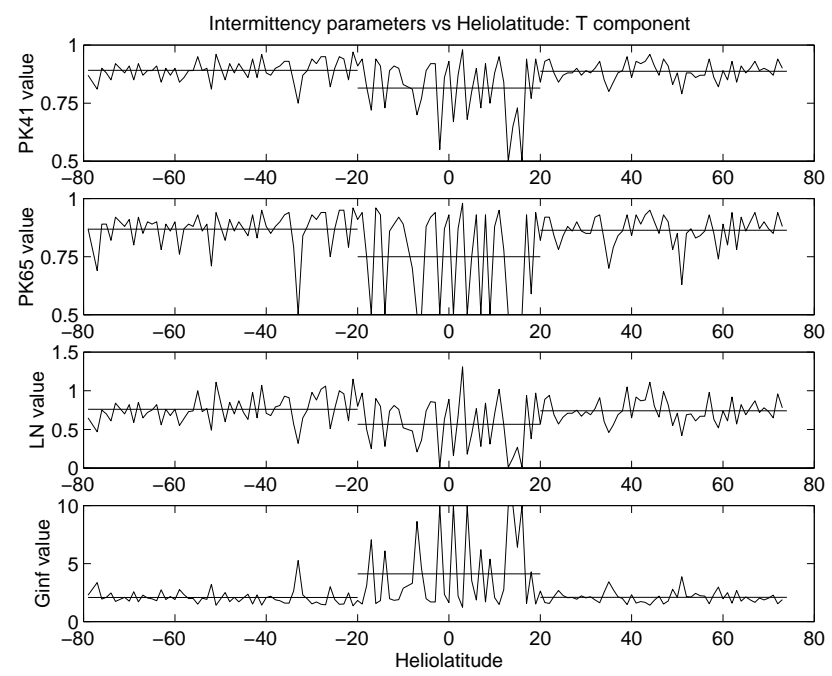

Fig. 7. Best-fit parameters for each of the models for the tangential component of the magnetic field plotted against heliolatitude. The horizontal lines represent the means in the different solar wind regions. Remember that higher G-infinity parameters corresponds to lower intermittency.
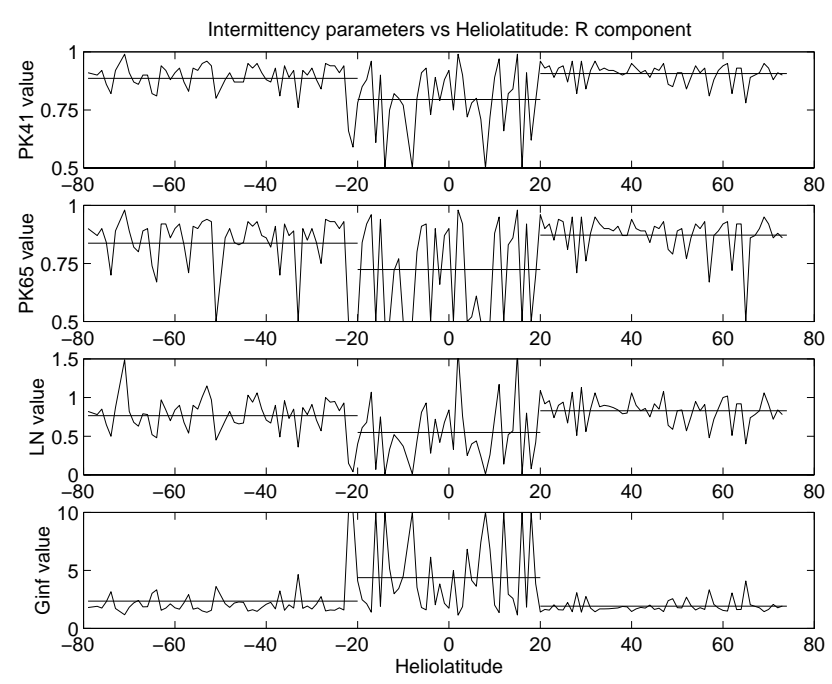

Fig. 8. Best-fit parameters for each of the models plotted against heliolatitude, for the radial component of the magnetic field. The horizontal lines represent the means in the different solar wind regions.

eter and the least-squares goodness of fit value.

The best-fit parameter for each model and its corresponding goodness of fit (in the least-squares sense) were plotted against the heliolatitude. Figures 7 and 8 show the best-fit parameters while Figs. 9 and 10 show the corresponding goodness of fit.

Again, the horizontal lines represent the parameter means in the distinct solar wind regions (see Tables 3 and 4 for actual values). The parameters in each case correspond to the p-value, $\mu$-value and $g(\infty)$ value in the best-fit predicted

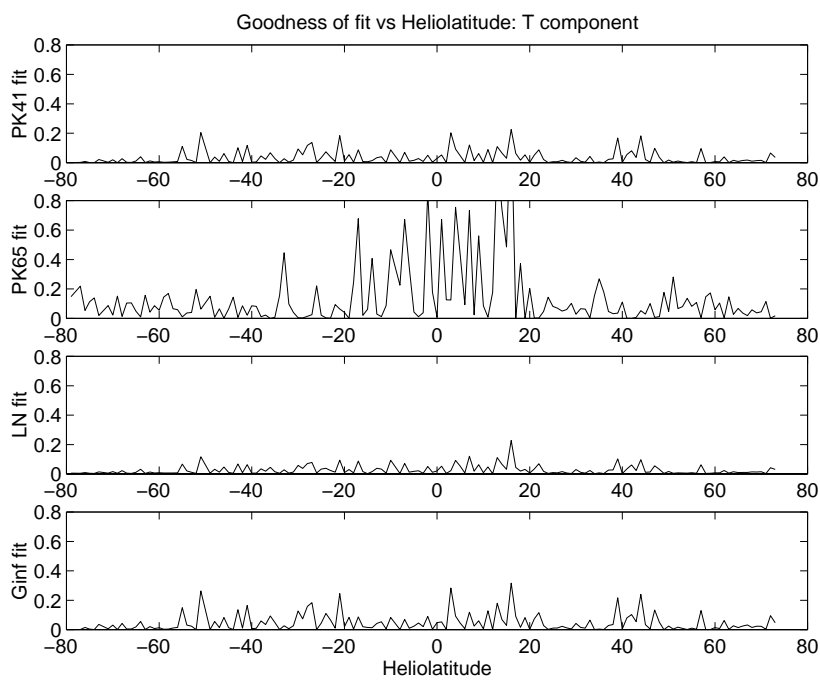

Fig. 9. Least-squares goodness of fit value for the models plotted against heliolatitude for the tangential component of the magnetic field.
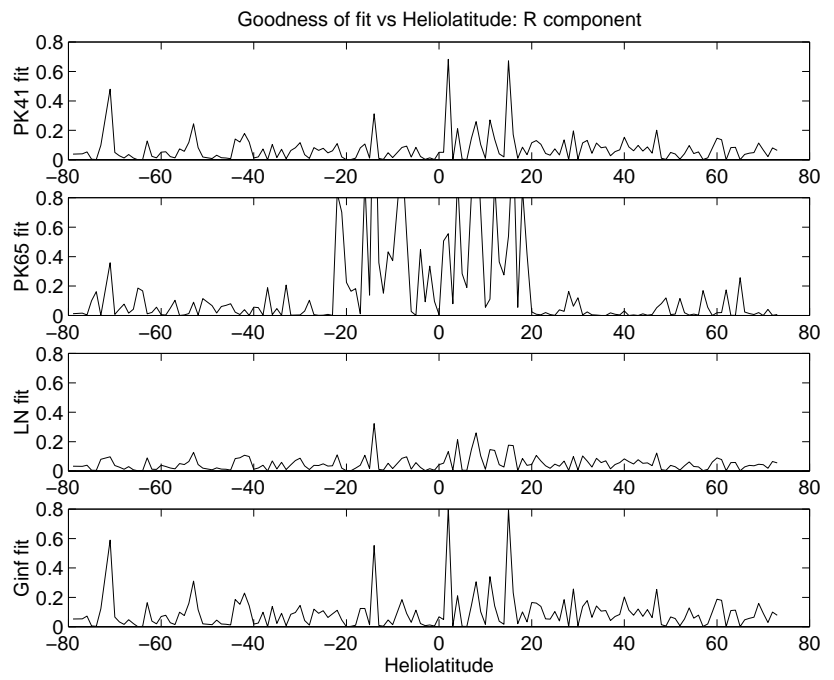

Fig. 10. Least-squares goodness of fit value for the models plotted against heliolatitude for the radial component of the magnetic field.

$g(q)$ curve. All the models show consistency across the radial and tangential components, and all indicate an increase in intermittency in the northern fast wind, but only for the radial component, an effect mirrored in the non-linear data suggesting it is a real phenomenon.

In all models, although less so in the lognormal one, the equatorial region is clearly distinguishable and the mean intermittency drops sharply, particularly in the P and G infinity models. The change is more marked than in the nonlinear analysis, and also the values in the fast wind are more steady. Certainly the goodness of fit in the equatorial region also changes, becoming markedly worse in the PK65 model. The lognormal and PK41 models seem least affected by the 
Table 3. Table of intermittency parameter means for the 4 models for the radial and tangential components of the solar wind magnetic field. $\mathrm{R}$ and $\mathrm{T}$ postscripts indicate radial and tangential component data, respectively. The three regions are southern solar wind, lowlatitude solar wind (equatorial region) and northern solar wind

\begin{tabular}{lccc}
\hline Parameter & S. Wind & Eq. Region & N. Wind \\
\hline PK41R & 0.89 & 0.79 & 0.91 \\
PK41T & 0.89 & 0.81 & 0.89 \\
PK65R & 0.84 & 0.72 & 0.87 \\
PK65T & 0.87 & 0.75 & 0.86 \\
LNR & 0.77 & 0.55 & 0.83 \\
LNT & 0.76 & 0.57 & 0.74 \\
GINFR & 2.4 & 4.4 & 1.9 \\
GINFT & 2.1 & 4.1 & 2.1 \\
\hline
\end{tabular}

change, but in general, there seem to be areas in the slow wind where intermittency drops to almost zero, although this is not mirrored as sharply in the non-linearity analysis, so we should be wary of these sudden drops.

In looking at the steadiness of the intermittency parameter in the fast wind, all the models except the PK65 one perform reasonably well, and all the models are unanimous in indicating a very high general level of intermittency. Again, to study the significance of these values and the changes in the slow wind, it is better to look at percentage changes in the means in the different regions (see Sect. 4 for details on how these are calculated) compared to the percentage variation over the whole range of latitudes (see Table 5), than to try to tease out any direct error values (especially since structure function indices often have very low direct errors, Horbury and Balogh, 1998). Similarly, the goodness of fit criteria do not have a direct impact on this variation, except in the PK65 model, where the fits becomes much worse in the equatorial region. We see that the mean drops are significant for all the models, except perhaps the lognormal model, which is on the borderline.

Marsch and Liu (1993) studied solar wind fluctuations in the inner heliosphere, looking at both proton velocity and Alfvén velocity. They point out that at these distances (up to $1 \mathrm{AU}$ ), solar wind turbulence is often not fully developed, whereas the turbulence assumed by intermittency models is fully developed. They assumed that their data were, however, still relevant to questions of intermittency. The data we present here is further from the sun than the Helios data they used, but the question of developed turbulence is still pertinent, and part of the reason for using high frequency data and restricting ourselves to a maximum $300 \mathrm{~s} \mathrm{time} \mathrm{lag} \mathrm{is} \mathrm{to} \mathrm{min-}$ imise the problems of using an undeveloped turbulent data set. Marsch and Liu (1993) found that the structure function analyses for the Alfvén velocity (and hence, the magnetic field) in the fast wind displayed less deviation from the Kolmogorov straight line than the same criteria in the slow wind, although they were only comparing a few sets of data. It should also be noted that our results deal with higher fre-
Table 4. Mean goodness of fit in the least-squares sense in each solar wind region for each model given to $2 \mathrm{dp}$

\begin{tabular}{lccc}
\hline Parameter & S. Wind & Eq. Region & N. Wind \\
\hline PK41R & 0.06 & 0.10 & 0.07 \\
PK41T & 0.03 & 0.05 & 0.03 \\
PK65R & 0.08 & 0.44 & 0.03 \\
PK65T & 0.08 & 0.31 & 0.07 \\
LNR & 0.04 & 0.07 & 0.05 \\
LNT & 0.02 & 0.04 & 0.02 \\
GINFR & 0.08 & 0.13 & 0.05 \\
GINFT & 0.05 & 0.06 & 0.04 \\
\hline
\end{tabular}

quencies than those of Marsch and Liu.

Tu et al. (1996) used an extended P model developed for undeveloped turbulence in order to study intermittency in magnetic field fluctuations, again using Helios data. They found that while the intermittency for solar wind velocity seemed similar in fast and slow speed streams, the same could not be said for the components of the magnetic field. Instead, they found that while the intermittency in the fast wind was relatively stable, the intermittency parameter for the magnetic components in the slow wind varied a great deal, from relatively low values to very high ones, most notably in the radial component. Even though they used only a few data sets (3 from slow wind, 2 from fast wind) and their structure function time lags extended over a greater period than ours (from $81 \mathrm{~s}$ to $2500 \mathrm{~s}$ ), we feel that the results presented here support those found by Tu et al. (1996).

Sorriso-Valvo et al. (1999) looked at bulk solar wind velocity and magnetic field intensity using Helios data to analyse the probability distribution functions (PDFs) of the fluctuations while investigating intermittency. They used time scales from $81 \mathrm{~s}$ to 1 day to analyse the data, and considered fast and slow streams separately, although all data originated from the equatorial region. They found scaling for the time interval region between $81 \mathrm{~s}$ and around $200 \mathrm{~s}$, and considered intermittency in this range, which falls within the structure function time ranges used here. Their results indicated that the intermittency in the magnetic field intensity was roughly the same for both slow and fast wind. It seems reasonable to conjecture that perhaps there is more than one type of 'slow wind', with one type having almost zero intermittency and the other types being as intermittent, if not more so, than the fast wind.

Ruzmaikin et al. (1995) studied fluctuations in the magnetic field using Ulysses data far from the Sun (at 3.9 AU), and concentrated on high frequency data with time scales from $60 \mathrm{~s}$ to $3600 \mathrm{~s}$. They also found a very high level of intermittency in the solar wind, but their data correlated well with the Kraichnan model of turbulence, unlike ours. However, Horbury and Balogh (1998) later argued that their results were suspect because of the high orders of structure functions used.

Marsch and Tu (1997) also looked at Alfvén velocity fluc- 
Table 5. Percentage variation of the means between fast and slow wind compared with the base variation across all heliolatitudes for intermittency parameters. Percentages have been rounded to the nearest integer

\begin{tabular}{lcc}
\hline Model & Variability (\%) & Diff. in Means $(\%)$ \\
\hline PK41R & 14 & 21 \\
PK41T & 11 & 16 \\
PK65R & 24 & 27 \\
PK65T & 19 & 24 \\
LNR & 14 & 15 \\
LNT & 13 & 14 \\
GINFR & 16 & 25 \\
GINFT & 13 & 22 \\
\hline
\end{tabular}

tuations using absolute structure functions, but with time lags of $1 \mathrm{~s}$ to $10^{5} \mathrm{~s}$, using Helios data at $0.87 \mathrm{AU}$, and found that intermittency appeared to increase at smaller scales. They fitted the $\mathrm{P}$ model to their data for velocity fluctuations, finding a p-value of $0.7-0.8$. While this cannot be directly compared with our results for the magnetic data, it does suggest that the solar wind is a highly intermittent medium.

Horbury et al. (1996) looked at fluctuations in the magnetic field components using Ulysses data, at a distance of 4 AU. They used absolute value structure functions to study the data, using time lags of $10 \mathrm{~s}$ to 2 days, but split these time scales into three sections, with their small-scale region encompassing time lags of $80 \mathrm{~s}$ to $320 \mathrm{~s}$, corresponding to the time lags considered in this study. They found that these small-scale fluctuations were "significantly intermittent across all components", agreeing with our results. Horbury and Balogh (1998) also used absolute structure functions to study magnetic field fluctuations with time lags from $20 \mathrm{~s}$ $-200 \mathrm{~s}$, using the Ulysses data set. They found that the P model fitted their data best, and found a p-value of about 0.8 for these high frequency observations. An earlier study by Horbury et al. (1997b) found again that $p \sim 0.8$.

Burlaga (1995) studied intermittent fluctuations in solar wind velocity and magnetic field magnitude at 8.5AU and at large time scales, with lags from $0.85 \mathrm{hrs}$ to $13.6 \mathrm{hrs}$, and found a p-value of about 0.7 . However, given the difference between his data and the data considered here, it is possible that his data was sampling fluctuations of a different nature compared to the high-frequency ones we are studying here. Thus, our results should not be compared directly with those of Burlaga.

In all, the values presented here for the various intermittency parameters show a higher mean value than those of previous studies, which may well be due to the high frequencies we are studying. Certainly this study has highlighted the latitudinal variations of the small-scale intermittent features of the solar wind magnetic field in greater detail than has been achieved before, using the Ulysses fast-latitude scan.

The values of $g(3)$ and $g(4)$ over the whole range of latitudes were also considered (see Fig. 11). On this basis, it

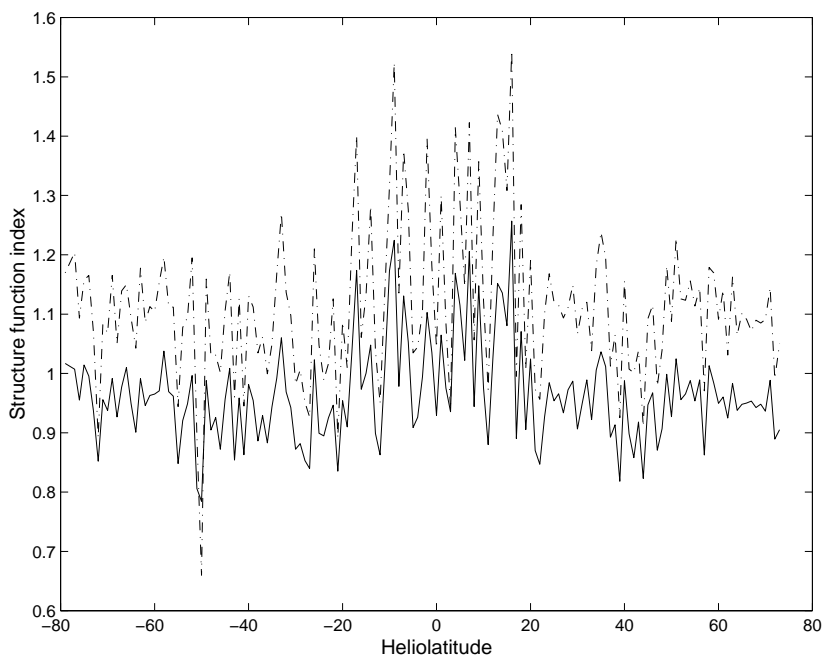

Fig. 11. Values of $g(3)$ and $g(4)$ vs. heliolatitude taken for time lags up to 300s. The dashed line corresponds to $g(4)$ and the solid one to $g(3)$.

seems that the Kolmogorov description is better since $g(3)$ is more consistently close to 1 , with the mean across all heliolatitudes and all components for $g(3)$ being $0.96 \pm 0.04$ and for $g(4)$ being $1.11 \pm 0.06$. Verma et al. (1996) looked at the question of which of the Kolmogorov or Kraichnan descriptions better suited the solar wind, and found that while it was difficult to decide definitively between them, there were certain areas in which the Kolmogorov predictions outperformed the Kraichnan ones. Horbury and Balogh (1998) also found that for the small-scales they considered, the Kolmogorov picture was again the better one. Since the PK65 model shows, in addition, the worst fits and the worst stability, we have disregarded it in further analysis (although, as will be mentioned below, the fits of the PK65 model are not independent of the $g(4)$ value, so its lack of stability is a more important criterion). However, we stress that this does not say anything about the possible validity of the Kraichnan pictures at other frequency ranges, or radial distances from the Sun.

Furthermore, it should be noted that the value of $g(3)$ affects the goodness of fit of the actual models to the data. In general, the $g(3)$ value is less than 1 over the range of the fast wind regions and more than 1 in the equatorial region. Tu et al. (1996) found that their extended intermittency model predicted values of $g(3)$ to be less than 1 for regions of undeveloped turbulence, so perhaps the low values in the fast wind are a sign that the turbulence there is less developed than in the slow wind. Velli and Prunetti (1997) found, in general, that turbulent fluctuations from the poles are often less developed than those of the equatorial region. This should be kept in mind when studying the goodness of fit data presented here, and perhaps it helps to explain the higher values of $g(3)$ in the equatorial region. However, we do not know why the values in this region are generally higher than 1 . To see how the deviation of $g(3)$ from 1 affects the goodness of fit values, 

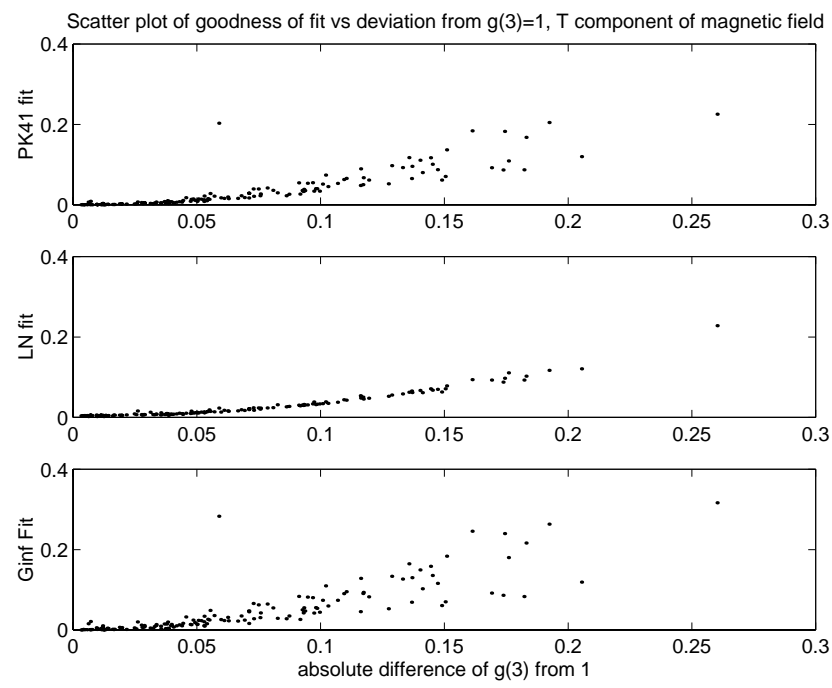

Fig. 12. This shows how the deviation of $g(3)$ from 1 affects the fit of the intermittency models.
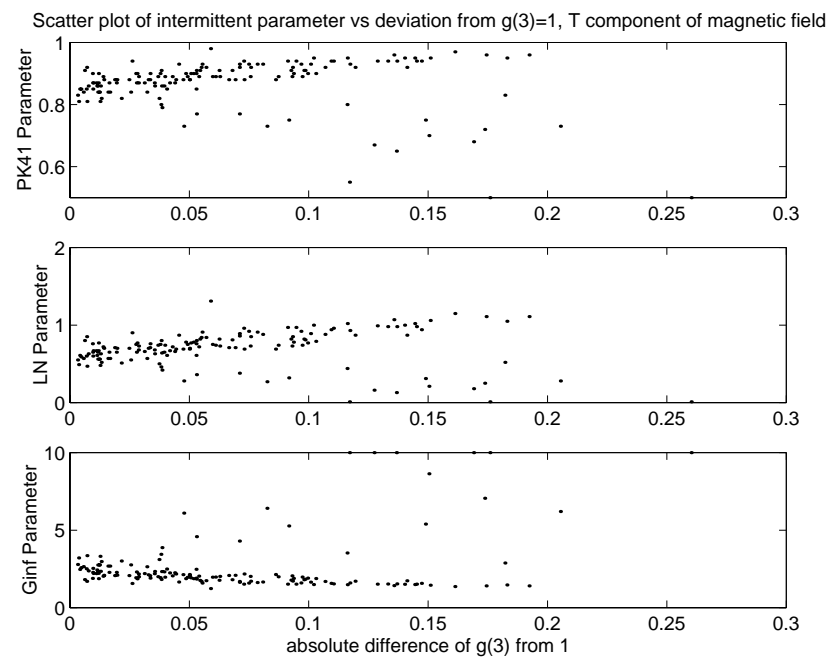

Fig. 13. This scatter plot shows how the deviation of $g(3)$ from 1 affects the parameter value of the intermittency models.

see Fig. 12.

It is clear that the fit of the models to the data is highly dependent on the value of $g(3)$. There is also a slight correlation between the $g(3)$ value and the actual best fit parameter value, as shown in Fig. 13, where the intermittency parameter tends to show a slightly greater degree of intermittency for larger deviations of $g(3)$ from 1 . This has also been shown by Horbury and Balogh (1998). However, there is no correlation between heliographic latitude and the deviation of $g(3)$, as shown in Fig. 14.

So, the differences shown in the parameter values in the different solar wind regions are still significant, although the influence of the $g(3)$ value should be noted. In particular, the strong dependence of goodness of fit on $g(3)$ means that it is very hard to distinguish between different models on the
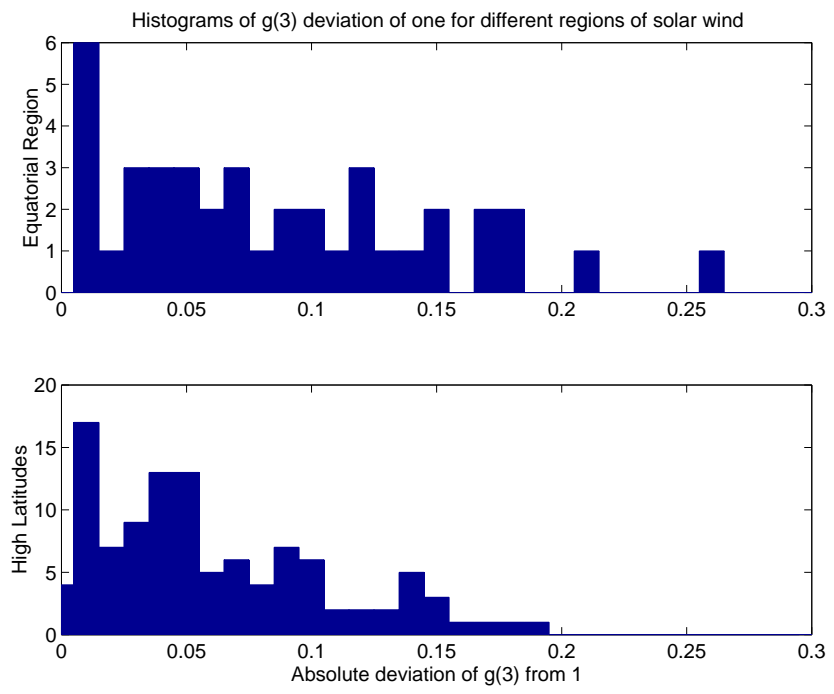

Fig. 14. The histograms here show the occurrence of deviations of $g(3)$ from 1 in the equatorial region (top plot), and at high latitudes (bottom plot). Here the equatorial region has been defined to run from $-20^{\circ}$ to $20^{\circ}$. Note that there are no significant differences between the two regions.

basis of their fits, since they all fit very well when $g(3) \sim$ 1. This may also explain the relatively poor fit of the PK65 model, since $g(4)$ is not close to 1 for much of the time, especially in the equatorial region.

\subsection{Extended self-similarity}

In both intermittent and non-intermittent turbulence, in the inertial range, the third order structure function should scale with the separation:

$S(3, \tau) \sim \tau$.

This relationship has led to the idea of extended selfsimilarity (ESS), where instead of plotting the structure functions against $\tau$, they are plotted against the third order structure function instead. So each index, $g(q)$, is worked out as the gradient of the log-scale plot of $S(q, \tau)$ vs. $S(3, \tau)$. This has been hypothesised to give an extended scaling regime for the time series, essentially extending the apparent inertial range. Plotting structure functions against the third order structure function, for velocity fluctuations, has been used to study the transition region between the small-scale inertial range and the dissipation range (Stolovitzky and Sreenivasan, 1993; Carbone, 1994; Sain and Bhattacharjee, 1999) and Grossmann et al. (1997) has also studied the scaling behaviour between the large-scale inertial range and the lowfrequency regime. Meneveau (1996) has also looked at the phenomenon of ESS, and is much more cautious about its abilities to extend the scaling regime in fluid turbulence. While most interest in ESS has been in neutral fluid turbulence, it has also been of interest in the solar wind turbulence (for instance, Carbone, 1994; Marsch and Tu, 1997). 


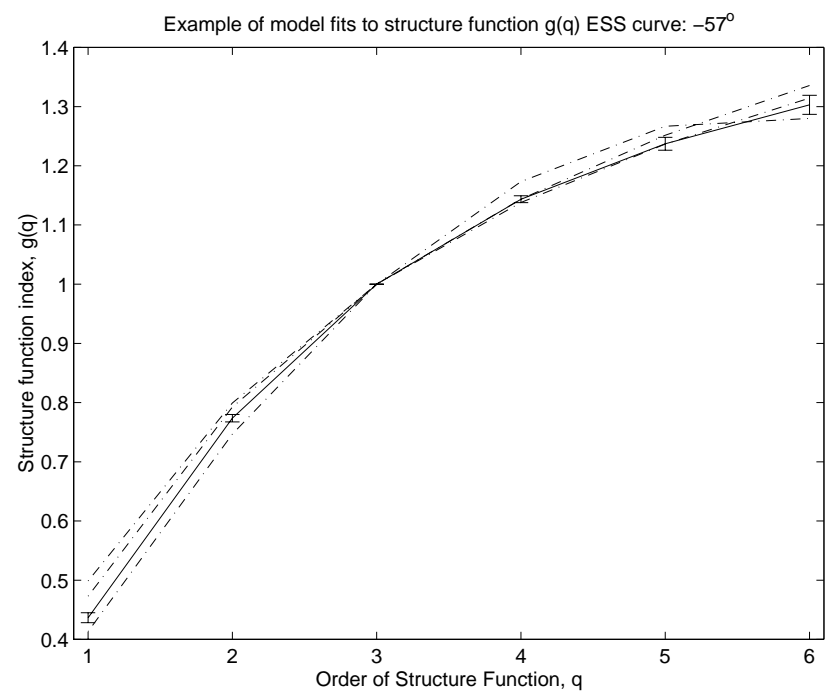

Fig. 15. The solid line represents the $g(q)$ curve obtained by using ESS on the data for $-57^{\circ}$ heliolatitude for structure functions with time lags $4 \mathrm{~s}$ to $300 \mathrm{~s}$. The dashed lines correspond to the three model fits (PK41, LN and Ginf) to this observed curve. The error bars in $g(q)$ are added as well.

We have used ESS to study the Ulysses fast-latitude scan data to see how fitting the models to the ESS $g(q)$ would compare with the results of the previous section. To look at the Kolmogorov picture, the structure functions were all plotted versus $S(3, \tau)$, the third order structure function, and their indices at that time taken for $q=1 \ldots 6$, for each degree of heliolatitude. We increased the order to 6 of the structure functions considered due to the better scaling obtained by using ESS (see below). Since it has been suggested that ESS gives a greater penetration into the dissipation range, a time lag of 4 seconds (the approximate order of the dissipation scale in the solar wind) was used to study the data instead of the 10 second time lag used when plotting the structure functions against $\tau$. The maximum time lag remained $300 \mathrm{~s}$. It should be noted that this proposed extension of the scaling regime could be due to the trivial $q / 3$ scaling in the dissipation region, which can be very similar to the structure function value in the inertial range (Stolovitzky and Sreenivasan, 1993). In terms of actually identifying intermittency, it does not matter which order of the structure function the others are plotted against, since any non-linearity would be visible regardless, but it is important in terms of fitting the models to the ESS results.

It is apparent that using ESS greatly improves the goodness of fit of the models to the data (see Fig. 15). However, the associated $g(q)$ error bars are also much smaller, so it is still meaningful to compare the goodness of fit values for the individual models. So, similar to the last section, the best-fit parameter was taken with its corresponding goodness of fit and plotted for each degree of heliolatitude (see Fig. 16).

Looking at Fig. 17 we see that the fits using ESS have improved by an order of magnitude. Given the relative size
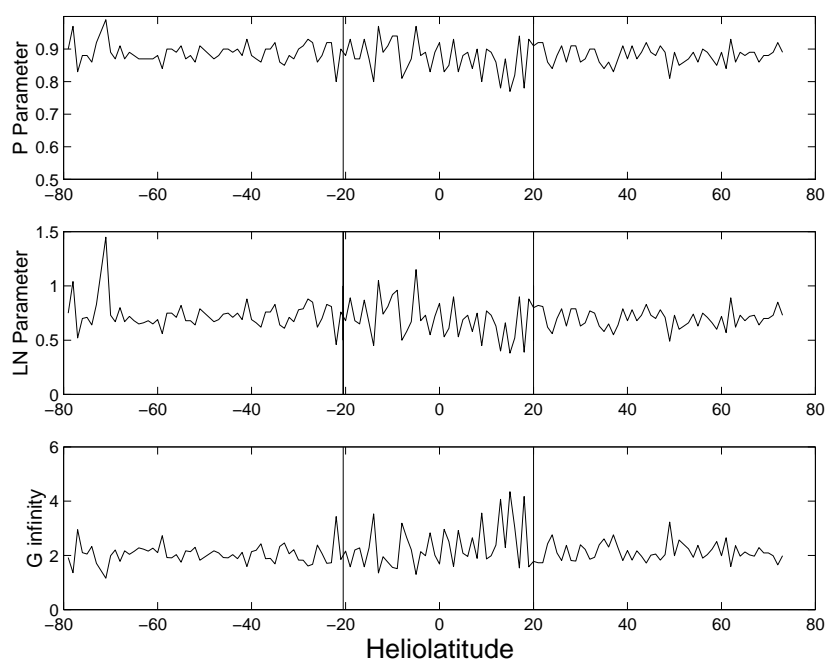

Fig. 16. Best-fit parameters of the models using ESS, where the vertical lines represent the approximate entries and exits into the equatorial region.
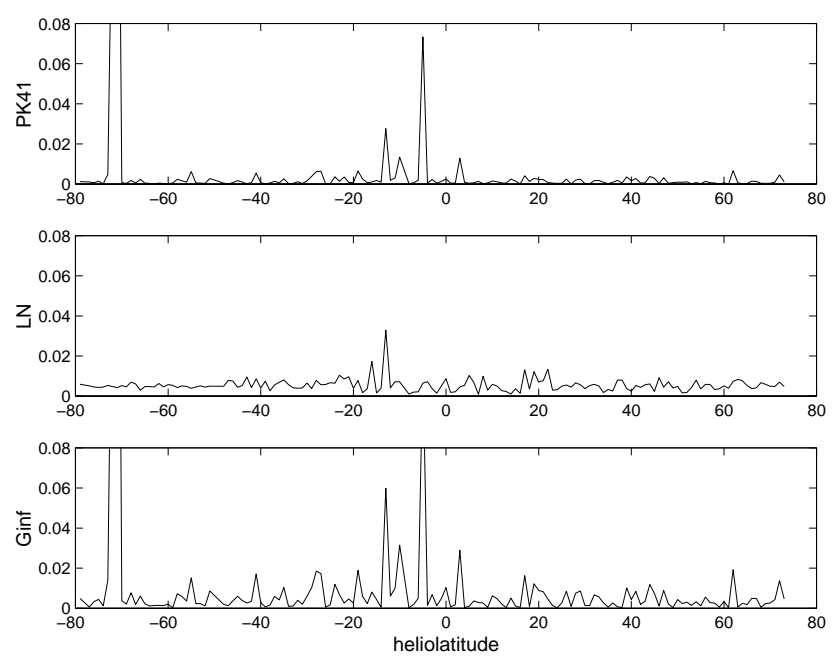

Fig. 17. The least-squares goodness of fit value of each of the three different models, using ESS analysis against heliolatitude.

of the error in $g(q)$, there is nothing to choose between them. Regarding the actual parameter values (see Fig. 16), one of the first things to notice is that the drop in intermittency in the equatorial region disappears, leaving only a heightened variability in its place (see Table 6 for mean values). All the parameters remain stable in the fast wind, and all still show a high level of intermittency in the flow, consistent with the results for time lag. However, the increased intermittency for the radial component in the northern fast wind has also disappeared which, given that this was evident in the nonlinear plot, is a significant omission.

Looking back at the correlation between the deviation of $g$ (3) from 1 and the goodness of fit of the models, it seems probable that the better fits shown here are due, largely to the automatic requirement of ESS that $g(3)=1$. Also, since 
Table 6. Table of ESS intermittency parameter means for the radial and tangential components of the solar wind magnetic field for the different solar wind regions

\begin{tabular}{cccc}
\hline Parameter & S. Wind & Eq. Region & N. Wind \\
\hline PK41R & 0.87 & 0.86 & 0.87 \\
PK41T & 0.89 & 0.87 & 0.88 \\
LNR & 0.67 & 0.66 & 0.67 \\
LNT & 0.73 & 0.70 & 0.70 \\
GINFR & 2.4 & 2.7 & 2.3 \\
GINFT & 2.1 & 2.3 & 2.3 \\
\hline
\end{tabular}

there was a slight correlation between $g(3) \neq 1$ and a high intermittency parameter, the fact that $g(3)=1$ might also explain the slightly lower values of intermittency parameter found in the fast wind regions with ESS. There does not seem to be any obvious explanation for the difference in mean values in the equatorial region. In the next section, we will try to discriminate between the two pictures of intermittency presented by the time lag analysis and the ESS analysis, but the ESS should not be preferred by virtue of its better fits given the dependence on $g(3)$.

\section{Comparison with solar wind speed}

In order to try to understand more about the intermittency in the solar wind, particularly the changes seen in the equatorial region, we compared the data with the solar wind speed (see Fig. 18). Direct comparison is not very fruitful, so we used a scatter plot of the data, for example, a scatter plot of the PK41 parameters against solar wind speed in Fig. 19. The difference between fast and slow wind is immediately apparent, with fast solar wind exhibiting a uniform high intermittency, whereas there is no discernible pattern for slow wind, and a greater range in the intermittent parameter value. To look at this in more detail, we divided the data into fast and slow wind regions, with the boundary chosen to be $600 \mathrm{~km} / \mathrm{s}$. For each region, the different model parameters were binned into twenty bins, and the resulting frequency histograms plotted, using results for both the radial and tangential components. This is illustrated in Figs. 20 and 21.

Again, the difference between the fast and slow winds is marked, with the fast wind displaying steady high intermittency in the magnetic field components, and the slow wind exhibiting generally lower values of intermittency but also a much greater range of values: this is particularly noticeable for the radial component, again probably because the changes in the equatorial region affect this component the most. Clearly, this is a sign that different processes are occurring in the fast and slow winds, and also an indication of the fact that the slow wind is not a single type of structure. Its origin is not well understood and these results indicate that there may be different types of slow wind giving rise to differing levels of intermittency.

The same histogram analysis was performed for the ESS

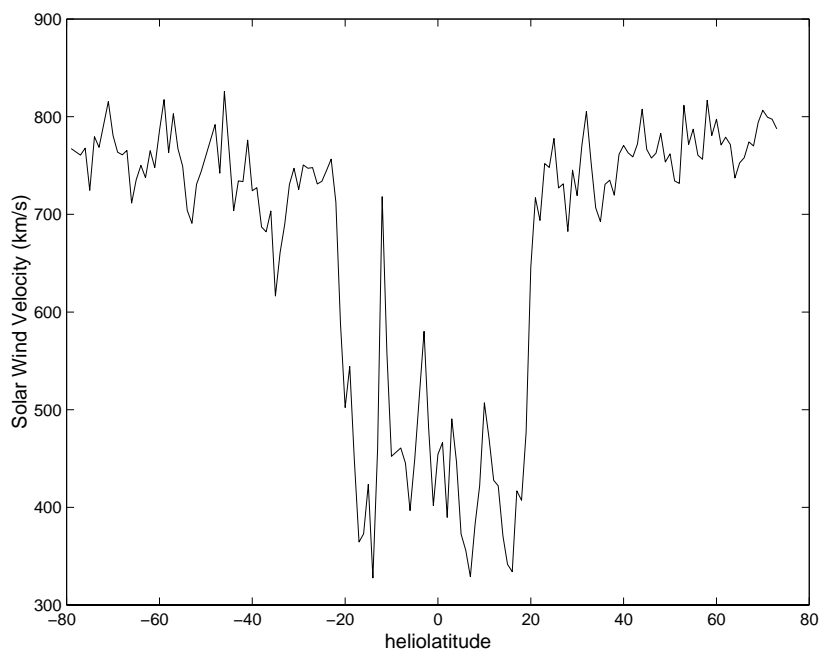

Fig. 18. Solar wind speed, averaged over each degree of latitude, plotted against heliolatitude.
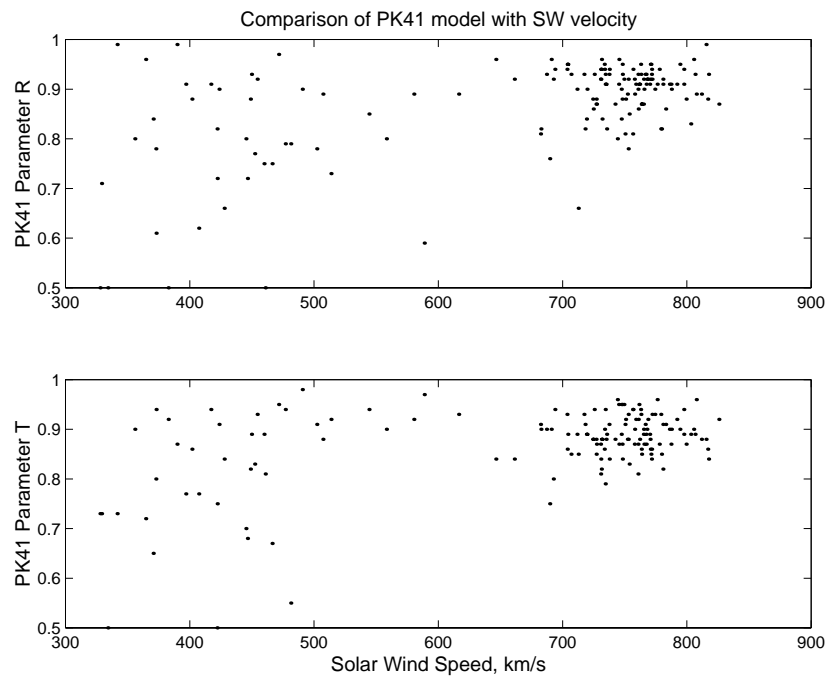

Fig. 19. Scatter plot of PK41 parameters for the radial and tangential magnetic field components vs. solar wind speed.

results (see Figs. 22 and 23) as a comparison. Certainly the differences seen for the time lag magnetic data between the fast and slow wind regions has all but disappeared, although the variability in the slow wind has remained, again most obviously for the radial component. The question is which of these two analyses, the time lag or the ESS, provide the best picture? To try to answer this question, we compared the histograms of the model parameters with the results from the non-linear analysis performed in the previous section. To do this across the different parameter ranges and models, all the histogram data was normalised, using $Y=(X-\mu) / \sigma$, where $\mu$ is the mean and $\sigma$ the standard deviation for each parameter in each region. These normalised histograms were then compared with the equivalent normalised histograms for the non-linear measure.

These comparisons are shown in Figs. 24, 25, 26 and 

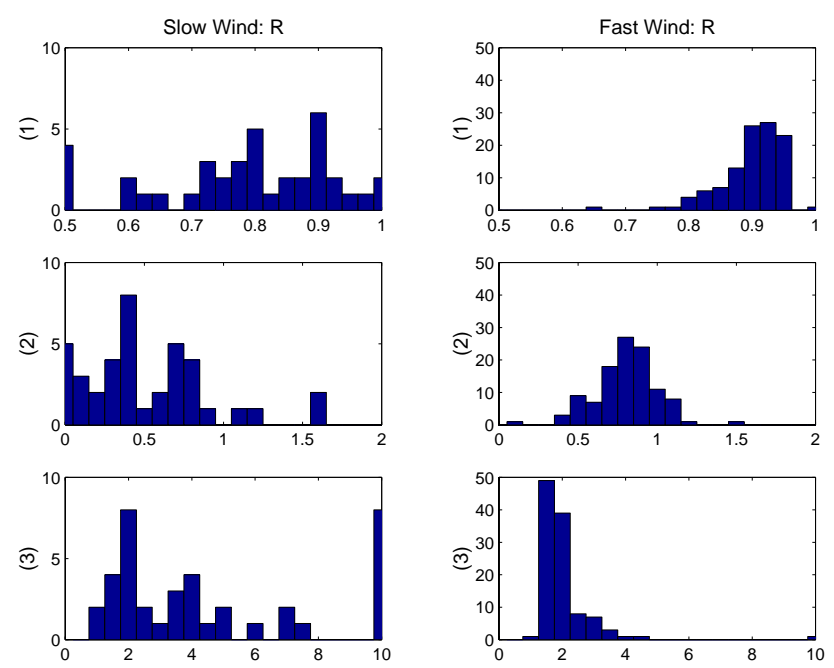

Fig. 20. Frequency histograms for intermittency parameters for the radial component of the magnetic field. (1) shows the PK41 modeli.e. the $x$-axis is the $\mathrm{P}$ parameter value and $y$-axis is the occurrence frequency in the two regions of solar wind, (2) shows Lognormal model (i.e. $\mu$ parameter) and (3) shows G-infinity model (i.e. the $g(\infty)$ parameter). The left-hand column represents data taken in the slow wind, and the right-hand column, data taken in the fast wind.
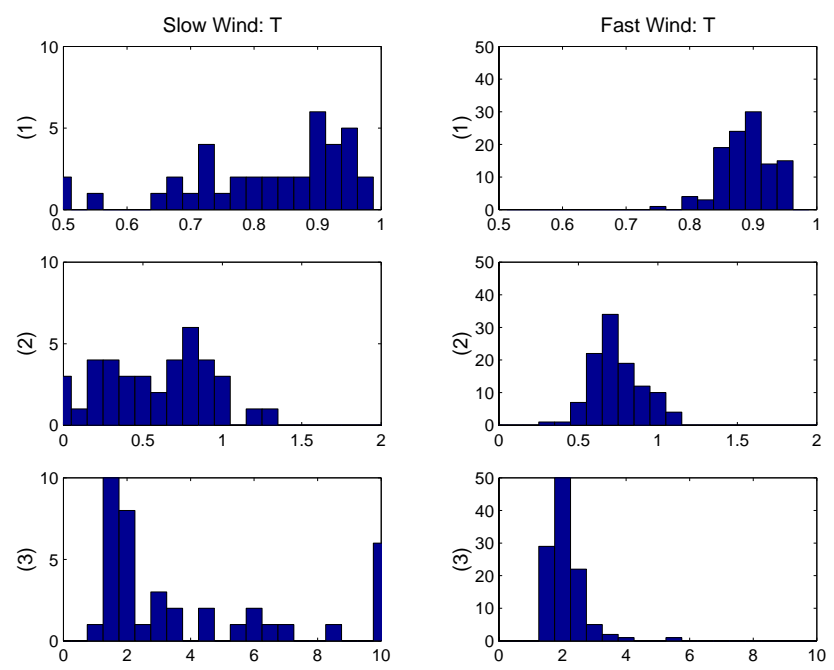

Fig. 21. Frequency histograms for the intermittent parameters for tangential component of the magnetic field. (1) shows the PK41 model, (2) shows Lognormal model and (3) shows G-infinity model. The left-hand column represents data taken in the slow wind, and the right-hand column, data taken in the fast wind.

27. One of the first points to be taken from these histograms is that the G-infinity model stops being as good a model of the processes taking place when these normalised histograms are considered, with its shape being very different to all the others. This is perhaps not surprising because it is precisely in this analysis that differences in the flow become important, since we are separating the data on the basis of flow velocity, and it is explicitly a model with no basis in any fluid physics.
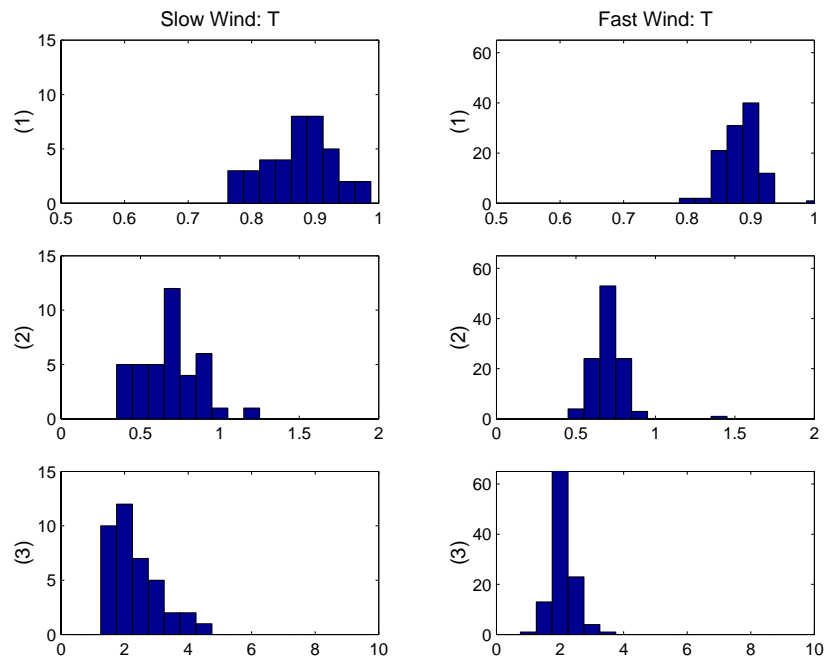

Fig. 22. Frequency histograms for the intermittent parameters for the tangential component of the magnetic field, obtained using ESS analysis. (1) shows the PK41 model, (2) shows Lognormal model and (3) shows G-infinity model. The left-hand column represents data taken in the slow wind, and the right-hand column, data taken in the fast wind.
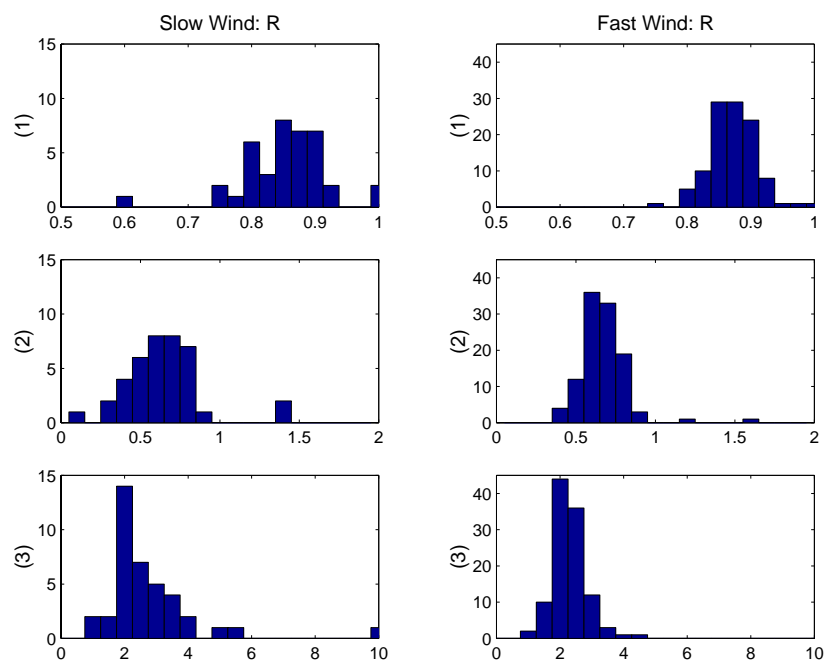

Fig. 23. Frequency histograms for the intermittent parameters for the radial component of the magnetic field, obtained using ESS analysis. (1) shows the PK41 model, (2) shows Lognormal model and (3) shows G-infinity model. The left-hand column represents data taken in the slow wind, and the right-hand column, data taken in the fast wind.

In comparing the four different figures, the lognormal model emerges as the best in mirroring the non-linear behaviour, and it is better at it (certainly for the T component) in the time lag analysis than the ESS. So, although ESS did demonstrate scaling down to 4 seconds, and hence into the dissipation region, it seems to remove too many features from the data to be a desirable method, especially since much of its increased 

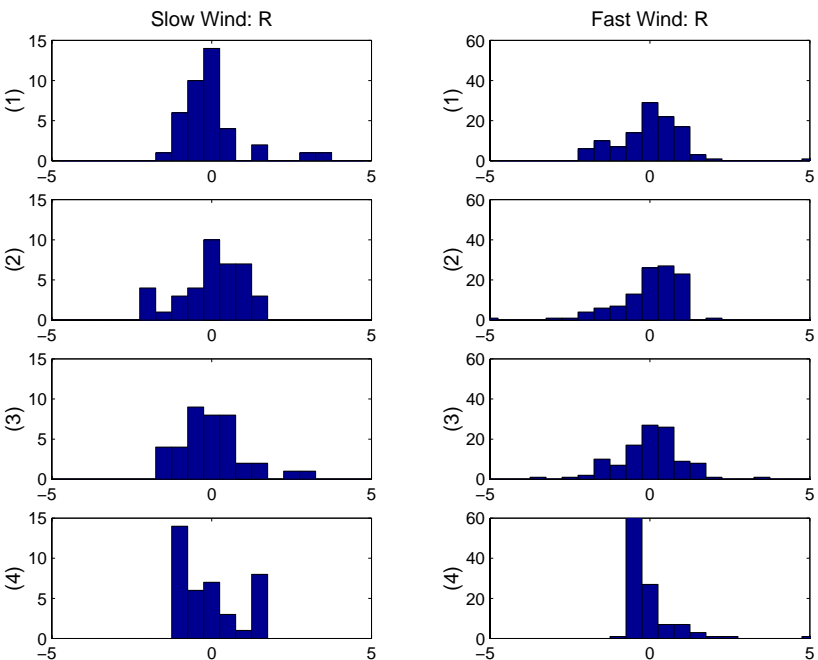

Fig. 24. Frequency histograms for the normalised intermittent parameters for the radial component of the magnetic field. (1) is the normalised histogram for the non-linear measure, (2) are normalised PK41 parameter values, (3) are normalised Lognormal parameter values and (4) are normalised G-infinity parameter values.
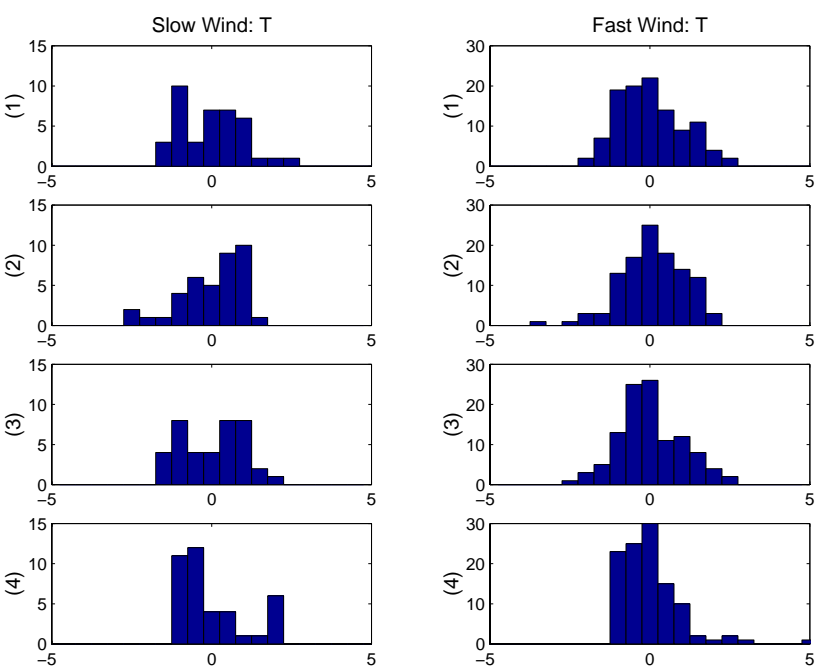

Fig. 25. Frequency histograms for the normalised intermittent parameters for the tangential component of the magnetic field. (1) is the normalised histogram for the non-linear measure, (2) are normalised PK41 parameter values, (3) are normalised Lognormal parameter values and (4) are normalised G-infinity parameter values.

goodness of fit may be based on the dependence of fit on $g(3)$, which is obviously fixed to be 1 in ESS methods.

\section{Discussion}

Ulysses' fast-latitude scan has given us the opportunity to study turbulent phenomena in the solar wind magnetic field over a large number of distinct time series, each correspond-
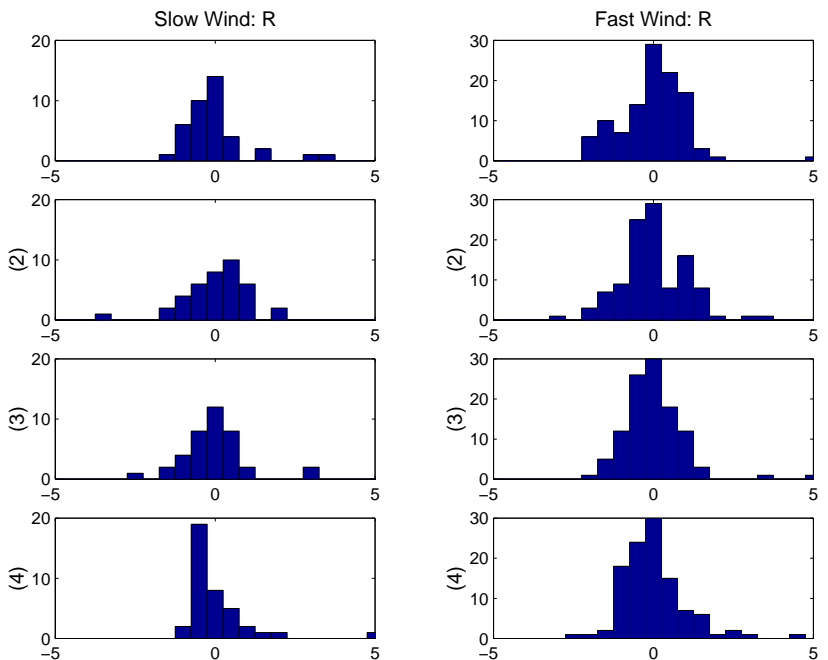

Fig. 26. Frequency histograms for the normalised intermittent parameters for the radial component of the magnetic field, using ESS analysis. (1) is the normalised histogram for the non-linear measure, (2) are normalised PK41 parameter values, (3) are normalised Lognormal parameter values and (4) are normalised G-infinity parameter values.
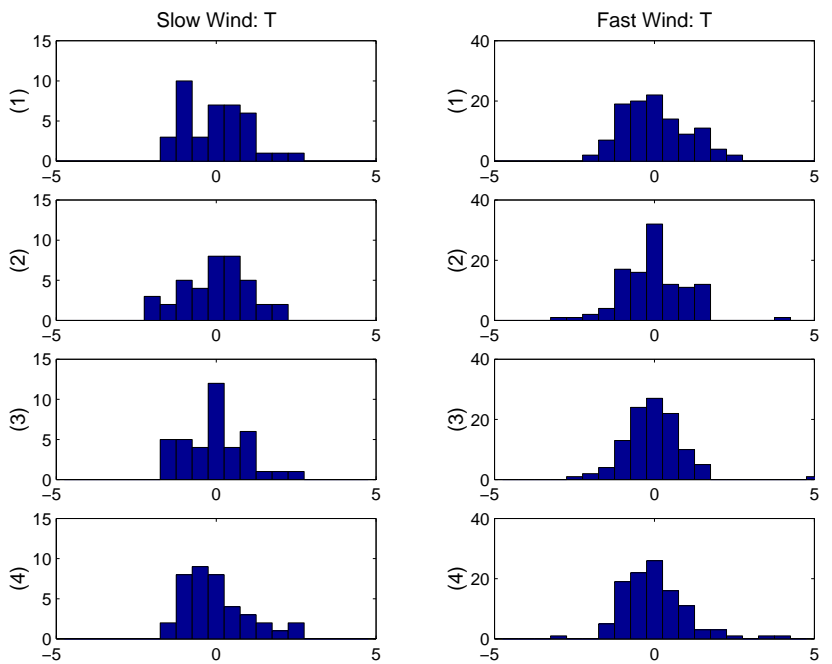

Fig. 27. Frequency histograms for the normalised intermittent parameters for the tangential component of the magnetic field, using ESS analysis. (1) is the normalised histogram for the non-linear measure, (2) are normalised PK41 parameter values, (3) are normalised Lognormal parameter values and (4) are normalised Ginfinity parameter values.

ing to a particular degree of heliolatitude. The spectral results corroborate previous work in identifying steady turbulence at high frequencies. It is clear from the results that the fast and slow wind show profoundly different properties, although the precise reasons for the steepening of the index in the equatorial regions is not known, and there may be some issues of stationarity in the slow wind data. The spectra presented here do, however, substantiate the view of the solar wind as 
a highly turbulent plasma, although we have studied only the magnetic field fluctuations.

Investigations into the nature of the magnetic field fluctuations showed that they were highly non-Gaussian and hence implied that the solar wind magnetic field was indeed intermittent. We concentrated on high frequency data, and found that, at least in the polar regions, the solar wind displayed a very high level of intermittency. However, the results from the equatorial region proved to be much more variable and difficult to interpret. In comparison with solar wind velocity, it seems clear that there exist different types of slow wind, with the model fits indicating various levels of intermittency, accounting for the variability in the equatorial region. There were significant sections of slow wind which displayed almost zero intermittency, leading to an average overall drop in intermittency in the central latitudes, while other sections displayed the same level of intermittency as the fast wind. Any future models of the slow wind will need to explain these variations.

In terms of the models used to evaluate the intermittency present in the solar wind, the lognormal and P model seem to provide the best explanation when the criteria of 'best fit', steadiness, and the non-linear measure are taken into account; however, as mentioned in Sect. 5.2, the goodness of fit criteria are affected by the $g(3)$ value. Since the lognormal model has performed so well, the relevance of the problems traditionally associated with it should be considered for the solar wind data. This has not yet been done. This gives some support to the concept of an inhomogeneous energy transfer model in the turbulent cascade.

The comparison of the models also gave us an opportunity to evaluate the Kolmogorov viewpoint against that of Kraichnan. The latter model is clearly the least well supported, simply by its poor fit with respect to the time lag, which is quite a fundamental criterion. When this is added to the lack of support from the comparison between $g(3)$ and $g(4)$ in Fig. 11, we conclude that the Kolmogorov picture of turbulence is more relevant to the solar wind at the frequencies and distances we were analysing.

Also, by studying the effect of the value of $g(3)$ on how well the models fitted the data, we saw that there was a close correlation with the goodness of fit and the nearness of $g(3)$ to its theoretical value of 1 . This relationship could easily account for the better fits obtained using ESS analysis. Certainly, on the basis of fits alone, it is not possible to discriminate between the models using ESS. Overall, considering also the ESS histogram results, the structure function analysis with respect to the time lag appears to better represent the data. It should be noted, however, that the question of the validity of ESS analysis in probing the transition regime has not been addressed in this paper.

Returning to the question of the multiplicative cascade models, the actual physical mechanisms on the solar wind which might give rise to such high values of $p$ or $\mu$ in the models are not known. Why 'eddies' in the solar wind would distribute energy so unevenly, or indeed, what the concept of an 'eddy' actually means in the context of the magnetic field, are questions which should be considered further, along with the question of how the phenomenological picture of the cascade actually relates to processes which might be occurring in the system.

The G-infinity model still performed remarkably well, given its origins. Its good fits could simply be a manifestation of the fact that we are trying to draw conclusions with curves fitted to only five data points, but its better performance than many fluid intermittency models (e.g. the SheLeveque and Kraichnan version of the P model) suggests a more profound reason. Burlaga, in his review on magnetohydrodynamics (Burlaga, 1995, p. 189), called it "remarkable" that the P model "derived to describe intermittent gas dynamic turbulence in a laboratory also describes MHD fluctuations on an astronomical scale" and inferred that "some universal scale-independent processes are involved", saying further that these must be "geometrical and kinematic". How much more astonishing then, that a model derived with no basis in fluid dynamics at all could provide such a good description. This may well indicate that there are universal, largely geometric, aspects to intermittency which dominate over the details of the system itself.

This study has also highlighted the limitations of structure functions for use in such an analysis. Clearly, the restriction to low orders makes accurate discrimination between different models difficult, and perhaps a different, more sensitive, data analysis approach is required. In the same vein, it would be useful to have an independent measure of intermittency in a data set, so that it can be unambiguously recognised and quantified from a time series, and the model predictions then could be compared with this measure, as opposed to the current situation where the model parameters can only really be compared with one another through their consistency, since even their relative goodness of fit becomes difficult to ascertain when the $g(3)$ effect is taken into account. The non-linear measure used in this paper is not a good enough measure for this purpose, but it is intended to demonstrate the sort of comparison that might be helpful. Possible measures might be kurtosis, or a singularity measure introduced by Davis et al. (1996) and Marshak et al. (1997).

It is certainly a quandary in space physics that solar wind turbulence seems so amenable to classical turbulent models, since it violates many of the assumptions inherent in these models; the solar wind is a compressible, supersonic, anisotropic and inhomogeneous flow. It has already been noted that the exponent function $g(q)$ is remarkably universal in terrestrial flows (Grossmann et al., 1997), so perhaps research into universal geometrical aspects of both turbulence and intermittency will move towards a resolution of this problem.

Acknowledgements. Ulysses work at Imperial College is sponsored by the UK Particle Physics and Astronomy Research Council, and C. Pagel is sponsored by a studentship from the UK Natural Environment Research Council. 


\section{References}

Balogh, A. and von Steiger, R., The heliosphere at solar minimum: Ulysses observations during its fast latitude scan in 1994-1995, Rev. Geophys., in press, 2001.

Balogh, A., Beek, T., Forsyth, R., Hedgecock, P., Marquedent, R., Smith, E., Southwood, D., and Tsurutani, B., The magnetic field investigation on the Ulysses mission: Instrumentation and Science, Astron. Astrophys. Supp., 92, 221-236, 1992.

Bavassano, B., Dobrowolny, M., Mariani, F., and Ness, N., Radial evolution of power spectra of interplanetary Alfvénic turbulence, J. Geophys. Res., 87, 3617-3622, 1982.

Belcher, J. and Davis, L., Large-amplitude Alfvén waves in the interplanetary medium, 2, J. Geophys. Res., 76, 3534-3563, 1971.

Borgas, M., A comparison of intermittency models in turbulence, Phys. Fluids A, 4, 2055-2061, 1992.

Bruno, R., Observations of MHD turbulence in the solar wind, Il Nuovo Cimento, 20C, 881-896, 1997.

Burlaga, L., Interplanetary Magnetohydrodynamics, Oxford University Press, Oxford, 1995.

Carbone, V., Cascade model for intermittency in fully developed magnetohydrodynamic turbulence, Phys. Rev. Lett., 71, 15461548, 1993.

Carbone, V., Scale similarity of the velocity structure functions in fully developed magnetohydrodynamic turbulence, Phys. Rev. E, 50, R671-R674, 1994.

Ching, E., Intermittency of temperature field in turbulent convection, Phys. Rev. E, 61, R33-R36, 2000.

Cho, J., Newell, R., and Sachse, G. W., Anomalous scaling of mesoscale tropospheric humidity fluctuations, Geophys. Res. Lett., 27, 377-380, 2000.

Coleman, P., Turbulence, viscosity and dissipation in the solar wind plasma, Astrophys. J., 153, 371-388, 1968.

Davis, A., Marshak, A., Wiscombe, W., and Cahalan, R., Scale invariance of liquid water distributions in marine stratocumulus. part I: Spectral properties and stationarity issues, J. Atmos. Sci., 53, 1538-1558, 1996.

Denison, D. G. T., Walden, A. T., Balogh, A., and Forsyth, R. J., Multitaper testing of spectral lines and the detection of the solar rotation frequency and its harmonics, Applied Statistics, 48, 4, 427-439, 1999.

Feynman, J. and Ruzmaikin, A., Distributions of the interplanetary magnetic field revisited, J. Geophys. Res., 99, 17 645-17651, 1994.

Frisch, U., Turbulence, Cambridge University Press, Cambridge, 1995.

Goldstein, M. and Roberts, D., Magnetohydrodynamic turbulence in the solar wind, Annu. Rev. Astron. Astrophys., 33, 283-325, 1995.

Goldstein, M. and Roberts, D., Magnetohydrodynamic turbulence in the solar wind, Phys. Plasmas, 6, 4154-4160, 1999.

Grossmann, S., Lohse, D., and Reeh, A., Application of extended self-similarity in turbulence, Phys. Rev. E, 56, 5473-5478, 1997.

Horbury, T., Ulysses Observations of Magnetic Field Fluctuations in the Heliosphere, Ph.D. thesis, Imperial College, London, 1996.

Horbury, T. and Balogh, A., Structure function measurements of the intermittent mhd turbulent cascade, Nonlinear Processes in Geophysics, 1998.

Horbury, T., Balogh, A., and Forsyth, R., Magnetic field signatures of unevolved turbulence in solar polar flows, J. Geophys. Res., 101, 405-413, 1996.
Horbury, T., Balogh, A., and Forsyth, R., The rate of turbulent evolution over the sun's poles, Astron. Astrophys., 316, 333-341, 1997a.

Horbury, T., Balogh, A., Forsyth, R., and Smith, E., Ulysses observations of intermittent heliospheric turbulence, Adv. Space Rev. 19, 847-850, 1997b.

Kolmogorov, A., The local structure of turbulence in incompressible viscous fluid for large Reynolds numbers, Proc. Roy. Soc. London, 434, 9-13, originally published in Dokl. Akad. Nauk. SSSR, volume 30, 301-305, 1991a.

Kolmogorov, A., Dissipation of energy in the locally isotropic turbulence, Proc. Roy. Soc. London, 434, 15-17, originally published in Dokl. Akad. Nauk. SSSR, volume 32, 16-28, $1991 \mathrm{~b}$.

Kraichnan, R., Inertial-range spectrum of hydrodynamic turbulence, Phys. Fluids, 8, 1385-1387, 1965.

Kraichnan, R., Models of intermittency in hydrodynamic turbulence, Phys. Rev. Lett., 65, 575-578, 1990.

Marsch, E. and Liu, S., Structure functions and intermittency of velocity fluctuations in the inner solar wind, Ann. Geophysicae, 11, 227-238, 1993.

Marsch, E. and Tu, C.-Y., Intermittency, non-Gaussian statistics and fractal scaling of mhd fluctuations in the solar wind, Nonlinear Processes in Geophysics, 4, 101-124, 1997.

Marshak, A., Davis, A., Wiscombe, W., and Cahalan, R., Scale invariance of liquid water distributions in marine stratocumulus. part II: multifractal properties and intermittency issues, J. Atmo. Sci., 54, 1423-1444, 1997.

Matthaeus, W. and Goldstein, M., Stationarity of magnetohydrodynamic fluctuations in the solar wind, J. Geophys. Res., 87, 10347-10354, 1982.

Matthaeus, W. and Goldstein, M., Low-frequency $1 /$ f noise in the interplanetary magnetic field, Phys. Rev. Lett., 57, 495-498, 1986.

Meneveau, C., Transition between viscous and inertial-range scaling of turbulence structure functions, Phys. Rev. E, 54, 36573663, 1996.

Meneveau, C. and Sreenivasan, K., The multifractal spectrum of the dissipation field in turbulent flows, Nuclear Physics B, Proceedings Supplement, 2, 49-76, 1987a.

Meneveau, C. and Sreenivasan, K., Simple multifractal cascade model for fully developed turbulence, Phys. Rev. Lett., 59, 1424 $1427,1987 \mathrm{~b}$

Percival, D. and Walden, A., Spectral Analysis for Physical Applications, Cambridge University Press, Cambridge, 1993.

Pierrehumbert, R., Anomalous scaling of high cloud variability in the tropical Pacific, Geophys. Res. Lett., 23, 1095-1098, 1999.

Politano, H. and Pouquet, A., Model of intermittency in magnetohydronamic turbulence, Phys. Rev. E, 52, 636-641, 1995.

Roberts, D., Klein, L., Goldstein, M., and Matthaeus, W., The nature and evolution of magnetohydrondynamic fluctuations in the solar wind: Voyager observations, J. Geophys. Res., 92, 11021 $11040,1987$.

Ruzmaikin, A., Feynmann, J., Goldstein, W., Smith, E., and Balogh, A., Intermittent turbulence in solar wind from the south polar hole, J. Geophys. Res., 100, 3395-3403, 1995.

Sain, A. and Bhattacharjee, J., Extended self-similarity and dissipation range of dynamics of three-dimensional turbulence, Phys. Rev. E, 60, 571-577, 1999.

Smith, E., Interplanetary magnetic field over 2 solar cycles and out to 20 AU, Adv. Space Res., 9, 159-169, 1989.

Smith, E., Balogh, A., Neugebauer, M., and McComas, D., Ulysses observations of Alfvén waves in the southern and northern solar 
hemispheres, Geophys. Res. Lett., 22, 3381-3384, 1995.

Sorriso-Valvo, L., Carbone, V., and Veltri, P., Intermittency in the solar wind turbulence through probability distribution functions of fluctuations, Geophys. Res. Lett., 26, 1801-1804, 1999.

Stolovitzky, G. and Sreenivasan, K., Scaling of structure functions, Phys. Rev. E, 48, R33-R36, 1993.

$\mathrm{Tu}, \mathrm{C}$. and Marsch, E., MHD Structures, Waves and Turbulence in the Solar Wind, Kluwer Academic Publishers, London, 1995.

Tu, C.-Y., Marsch, E., and Rosenbauer, H., An extended structurefunction model and its application to the analysis of solar wind intermittency properties, Ann. Geophysicae, 14, 270-285, 1996.

Vahnstein, S., Sreenivasan, K., Pierrehumbert, R., Kashyap, V., and Juneja, A., Scaling exponents for turbulence and other ran- dom processes and their relationships with multifractal structure, Phys. Rev. E, 50, 1823-1835, 1994.

Velli, M. and Prunetti, F., Alfvén waves in the solar corona and solar wind, Plasma Physics and Controlled Fusion, 39, B317-B324, 1997.

Veltri, P., MHD turbulence in the solar wind: self-similarity, intermittency and coherent sturctures, Plasma Physics and Controlled Fusion, 41, A787-A795, 1999.

Verma, M., Roberts, D., Goldstein, M., Ghosh, S., and Stribling, W., A numerical study of the nonlinear cascade of energy in magnetohydrodynamic turbulence, J. Geophys. Res., 101, 21619 $21625,1996$. 